\title{
GLI3 Processing by the Primary Cilium Regulates Muscle Stem Cell Entry into $\mathrm{G}_{\text {Alert }}$
}

\author{
Caroline E. Brun ${ }^{1,2}$, Marie-Claude Sincennes ${ }^{1,2}$, Alexander Y.T. Lin ${ }^{1,2}$, Derek Hall ${ }^{1,2}$, \\ William Jarassier ${ }^{4}$, Peter Feige ${ }^{1,2}$, Morten Ritso ${ }^{1,2}$, Fabien Le Grand ${ }^{4}$ and \\ Michael A. Rudnicki ${ }^{1,2,3, *}$
}

${ }^{1}$ Sprott Centre for Stem Cell Research, Regenerative Medicine Program, Ottawa Hospital Research Institute, Ottawa, ON, K1H 8L6, Canada

2 Department of Cellular and Molecular Medicine, Faculty of Medicine, University of OttawaOttawa, ON, K1H 8M5, Canada

${ }^{3}$ Department of Medicine, Faculty of Medicine, University of Ottawa, Ottawa, ON, K1H 8M5, Canada

${ }^{4}$ Institut NeuroMyoGène, Université Claude Bernard Lyon 1, CNRS UMR 5310, INSERM U1217, 69008 Lyon, France

*Corresponding Author (M.A.R., Email: mrudnicki@ohri.ca)

\begin{abstract}
Satellite cells are required for the growth, maintenance, and regeneration of skeletal muscle. Quiescent satellite cells possess a primary cilium, a structure that regulates the processing of the GLI family of transcription factors. Here we find that GLI3, specifically, plays a critical role in satellite cell activation. Primary cilia-mediated processing of GLI3 is required to maintain satellite cells in a $G_{0}$ dormant state. Strikingly, satellite cells lacking GLI3 enter $G_{\text {Alert }}$ in the absence of injury. Furthermore, GLI3 depletion or inhibition of its processing stimulates symmetrical division in satellite cells and expansion of the stem cell pool. As a result, satellite cells lacking GLI3 display rapid cell-cycle entry, increased proliferation and augmented self-renewal, and markedly enhanced long-term regenerative capacity. Therefore, our results reveal an essential role for primary cilia processing of GLI3 in regulating muscle stem cell activation and fate.
\end{abstract}




\section{INTRODUCTION}

Adult muscle stem cells, or satellite cells, reside within their niche between the basal lamina and myofiber sarcolemma in a reversible $G_{0}$ quiescent state ${ }^{1}$. Upon muscle damage, the niche is modified, inducing the transition from quiescence to activation in satellite cells. This process triggers mechano-property changes, migration, metabolic activation, increased RNA transcription and protein synthesis and cell cycle entry ${ }^{2-6}$. Once activated, satellite cells proliferate extensively to generate myogenic progenitors that differentiate and fuse to repair the injured myofibers, while a subset of satellite cells self-renew and return to quiescence to replenish the stem cell pool ${ }^{7,8}$. Hence, the ability to self-renew and reversibly enter quiescence is a hallmark of satellite cells that ensures proper muscle repair throughout life. Interestingly, in response to extrinsic cues, satellite cells can dynamically transit from a $\mathrm{G}_{0}$ to a $\mathrm{G}_{\text {Alert }}$ quiescent state, which is mediated by mTORC1 signaling ${ }^{6,9-11}$. Whether other pathways regulate this poised activation state that confers enhanced regenerative capacity to the $\mathrm{G}_{\text {Alert }}$ satellite cells remains unestablished.

A high proportion of quiescent satellite cells harbor a primary cilium, which rapidly disassembles upon activation and reassembles preferentially in self-renewing satellite cells ${ }^{12}$. The primary cilium is a small, non-motile, microtubule-based structure anchored to a cytoplasmic basal body that protrudes from cells in $\mathrm{G}_{0}{ }^{13}$. It acts as a nexus for cellular signaling, most notably Hedgehog signaling ${ }^{14,15}$, wherein cilia-mediated processing of the GLI-family of transcription factors is required for Hedgehog signal transduction ${ }^{16-18}$. Although there are three GLI transcription factors (GLI1-3), only GLI3 contains a potent N-terminal repressor domain.

In the absence of Hedgehog ligands, GLI3 is sequentially phosphorylated at the base of the cilium, first by the cAMP-dependent protein kinase $A$ (PKA) and then by glycogen synthase kinase 3 (GSK3) and casein kinase 1 (CK1) ${ }^{19-23}$. GLI3 phosphorylation promotes its proteolytic cleavage, converting it to a repressor form. Accordingly, decreased PKA activity leads to the activation of Hedgehog signaling independently of Hedgehog ligand-receptor binding ${ }^{24-26}$. Hedgehog ligandreceptor binding induces the accumulation of GLI3 at the cilium tip, thereby limiting its phosphorylation and cleavage ${ }^{16,21}$. Thus, the primary cilium controls the balance between the fulllength activator (GLI3FL) and cleaved repressor (GLI3R), which typically dictates the activation state of Hedgehog signaling.

Although studies have demonstrated that primary cilia act as a signaling hub, their role in satellite cell function remains unknown. Here, we identify GLI3 as a mediator of the cellautonomous, cilia-related control of satellite cell activation. Using a conditional knockout strategy, we show that ciliary processing of GLI3 to the repressor form (GLI3R) controls the $\mathrm{G}_{0}$ to $\mathrm{G}_{\text {Alert }}$ transition of quiescent muscle stem cells through regulation of mTORC1 signaling, independent of canonical Hedgehog downstream target gene expression. Moreover, we find that GLI3R regulates satellite cell commitment by promoting asymmetric cell division and that genetic ablation of Gli3 promotes symmetric cell expansion and improves muscle repair. We conclude that primary cilia-mediated GLI3 processing controls the activation and regenerative capacity of muscle stem cells. Furthermore, we identify GLI3 as a potential therapeutic target to promote muscle stem cell engraftment in conditions such as Duchenne muscular dystrophy. 


\section{RESULTS}

\section{GLI3 is the major GLI-family transcription factor expressed in muscle stem cells}

Satellite cells harbor a primary cilium ${ }^{12,27,28}$, yet the cilia-mediated pathways regulating satellite cell function remain uncharacterized. As the Hedgehog pathway requires the primary cilium for its transduction ${ }^{14}$, we hypothesized that cilia-mediated Hedgehog signaling regulates satellite cell function.

We first performed RNA-sequencing on freshly isolated satellite cells from resting and injured muscles. Although FACS method induces partial activation ${ }^{29-31}$, we refer to the freshly isolated satellite cells as quiescent satellite cells (QSCs) in the manuscript. In addition, the mixed population of proliferating satellite cells and progenitors isolated from 3 days post-cardiotoxin (CTX)-injured muscles will be referred as to activated satellite cells (ASCs ${ }^{32}$. We observed that the components of canonical Hedgehog signaling, namely the receptor Patched1 (Ptch1), the signal transducer Smoothened (Smo) and the three transcriptional effectors Gli1, Gli2 and Gli3, are expressed in quiescent satellite cells (Supplementary Fig. 1a). However, expression of conserved GLI-target genes ${ }^{33}$, such as Gli1, Ptch1, Ptch2, Hhip and Bcl2, is significantly downregulated as satellite cells transit from quiescence to activation (Supplementary Data file 1; Supplementary Fig. 1b). The increased expression of Cdk6, Ccnd2 and Ccnd1 is likely due to the cycling state of the ASCs. Together, these results indicate that canonical Hedgehog signaling is turned off as QSCs become activated. Of note, the transcriptional effectors Gli1 and Gli2 are strikingly downregulated, while Gli3 expression is significantly increased in ASCs (Supplementary Data file 1; Supplementary Fig. 1b). RT-qPCR on QSCs, ASCs, proliferating myoblasts and differentiated myotubes further confirmed that only Gli3 is enriched in ASCs and proliferating myoblasts (Supplementary Fig. 1c).

\section{Primary cilia dynamically regulate GL/3 proteolytic processing in myogenic cells}

As GLI3 activity relies on the primary cilium ${ }^{17,21}$, we investigated the kinetics of primary cilia assembly and disassembly on cultured myofibers and primary myoblasts. Primary cilia were labelled by immunostaining using antibodies directed against the primary cilium-specific protein ARL13B and acetylated $\alpha$-TUBULIN (acaTUB), and basal bodies were stained with $\gamma$-TUBULIN (YTUB) (Fig. 1; Supplementary Fig. 1d, e). In line with previous observations ${ }^{12}$, we found that more than $60 \%$ of the satellite cells on freshly myofibers isolated from extensor digitorum longus (EDL) muscle have a primary cilium that disassembles during proliferation and reassembles specifically in the PAX7+ self-renewing cells (Fig. 1b). As well, proliferating myoblasts are rarely ciliated and only the $\mathrm{PAX} 7^{+}$'reserve' cells ${ }^{34}$ reassemble a primary cilium upon differentiation (Fig. 1d).

Full-length and non-PKA phosphorylated GLI3 (GLI3FL) localizes in the primary cilium, while the cleaved repressor GLI3R is found at the basal body of the cilium where its proteolytic processing occurs ${ }^{16,21,35,36}$. To confirm that similar regulation occurs in myogenic cells, we treated primary myoblasts with SAG, a SMOOTHENED agonist that induces accumulation of GLI3FL in the primary cilium, thus abrogating its proteolytic processing ${ }^{21,36}$ (Supplementary Fig. 2a-g). Consequently, SAG-induced Hedgehog stimulation decreases the level of GLI3R, leading to the upregulation of the two well-known GLI-target genes, Gli1 and Ptch1 (Supplementary Fig. 2a-f). To assess the effects of negative regulation, myoblasts were treated with forskolin (FSK), an adenylyl cyclase activator that stimulates $\mathrm{PKA}^{21,35}$. FSK treatment prevents GLI3 ciliary 
accumulation, promotes GLI3 proteolytic cleavage and Gli1 and Ptch1 expression is decreased (Supplementary Fig. 2a-e, g). Therefore, we conclude that cilia and PKA-mediated regulation of GLI3 processing is conserved in myogenic cells.

We then assessed the temporal coordination of muscle cell ciliation and both GLI3 localization and proteolytic processing by immunostaining and Western blot. In ciliated QSCs on freshly isolated myofibers (T0), GLI3 antibody clearly labels the basal body, where PKA is expressed (Fig. 2a), suggesting that GLI3 is expressed as a repressor. As satellite cells activate and lose primary cilia, GLI3 localizes in the cytoplasm and around the microtubule-organizing centers (Fig. 2a). In cultured primary cells, although primary ciliation is rare due to their proliferative activity, $65 \%$ of ciliated myoblasts display GLI3 accumulation at the axoneme and the tip of primary cilia (Fig. 2b, c). In non-ciliated myoblasts, GLI3 is mainly found in the cytoplasm, as observed in ASCs on myofibers (Fig. 2a, b). GLI3FL protein levels are the highest in proliferating myoblasts and decrease during myogenic differentiation concomitantly with the ratio of GLI3FL/GLI3R (Fig. 2d-g), which correlates with the kinetics of ciliation and ARL13B expression (Fig. 2b-g). Finally, as myoblasts undergo differentiation, GLI3 progressively transits from the axoneme to the basal body of primary cilia and at $72 \mathrm{~h}$, most of the PAX7+ 'reserve' cells have a cilium and maintain high GLI3R, whereas myotubes have no primary cilium and do not express GLI3 (Fig. 2).

In the absence of ligand stimulation, primary cilia trigger GLI3 proteolytic processing, restraining the Hedgehog pathway in an off state ${ }^{13}$. To assess whether primary cilia-mediated processing of GLI3 controls the output of Hedgehog signaling in myogenic cells, primary myoblasts were treated with either siRNA targeting Gli3, or with siRNA against Ift88 to disrupt primary cilia assembly and consequently GLI3 processing ${ }^{18,37}$ (Supplementary Fig. 2h-I). Knocking-down Ift88 decreases GLI3R levels, consequently increasing GLI3R/GLI3FL ratio and Gli1 and Ptch1 expression (Supplementary Fig. 2h-k). Interestingly, Gli3 siRNA treatment induces similar upregulation of the two GLI-target genes (Supplementary Fig. 2I), indicating that GLI3FL is dispensable for GLI-target gene expression and that the predominant regulatory form is the repressor.

These results collectively show that GLI3 is processed to a repressor in ciliated QSCs. Transient primary cilia disassembly during cell cycle entry and proliferation abolishes GLI3R processing, promoting ciliary GLI3FL accumulation. Upon differentiation, myoblasts reassemble a primary cilium that will be only maintained in the self-renewing 'reserve' cell population, which then expresses GLI3R at the base of the cilium. Thus, our results demonstrate that the ratio of GLI3FL/GLI3R relies on cilia dynamics and is regulated independently of Hedgehog exogenous signals as muscle stem cells progress through the myogenic lineage.

\section{Loss of GLI3R does not affect Hedgehog signaling in satellite cells}

To better characterize the role of GLI3R in regulating satellite cell function, we generated

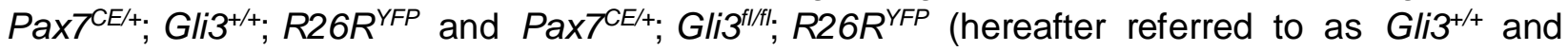
Gli3 $^{\Delta \Delta}$ mice, respectively), in which Gli3 was excised in satellite cells that are simultaneously labelled by YFP upon tamoxifen induction (Supplementary Fig. 3a). The efficiency of Gli3 excision was confirmed in QSCs and their myogenic descendants (ASCs, MBs and MTs) (Supplementary Fig. 3b, c). 
Uninjured resting muscle exhibit no gross histological abnormalities upon tamoxifen treatment (Fig. 3a). However, Gli3 ${ }^{\Delta / \Delta}$ muscles were found to have a transient increase in the number of satellite cells following tamoxifen injections (Fig. 3b). This observation led us to hypothesize that Gli3 deletion in satellite cells could be inducing a break from quiescence. During homeostasis, satellite cells reside in their niche, which maintains their quiescence, but then migrate outside the basal lamina upon activation ${ }^{2,5}$. Analyzing muscle cross-sections and myofibers post-tamoxifen injection showed that both $\mathrm{Gli3}^{+/+}$and Gli3 ${ }^{\mathrm{N} \Delta}$ satellite cells are located in their niche, underneath the basal lamina (Supplementary Fig. 3d-f) and that Gli3 ${ }^{+/+}$and Gli3 ${ }^{\Delta / \Delta}$ mice exhibit similar proportion of ciliated cells (Fig. 3c, d). Together, these results indicate that $\mathrm{Gli}^{\mathrm{\Delta} \Delta}$ satellite cells are inherently in quiescent state and suggests that Cre-mediated Gli3 deletion transiently activates satellite cells, increasing their number over weeks after tamoxifen treatment.

To further characterize the consequences of Gli3 deletion, we performed RNA-sequencing on Gli3 $^{+/+}$and Gli3 ${ }^{\Delta / \Delta}$ QSCs and ASCs and compared their transcriptome (Fig. 3e; Supplementary Fig. $\mathbf{3 g}$ ). Principle component analysis (PCA) of the transcriptional profiles of $\mathrm{Gli3}^{+/+}$and Gli3 ${ }^{\Delta / \Delta}$ SCs revealed two distinct groups based on the first component axis (PC1), distinguishing the QSCs from the ASCs (Fig. 3e). PCA also revealed a high correlation between $\mathrm{Gli}^{+/+}$and $\mathrm{Gli}{ }^{\mathrm{N} \Delta}$ QSCs, corroborating the idea that the Gli3 ${ }^{\Delta / \Delta}$ SCs are predominantly quiescent. Interestingly, the correlation between the QSC and ASC transcriptional signatures was slightly increased in the Gli3 ${ }^{\Delta / \Delta}$ background (0.67) than the Gli3 $^{+++}(0.66)$ (Fig. 3e), suggesting that the Gli3 $^{\Delta \Delta}$ QSCs may actually exhibit some features of satellite cell activation.

Surprisingly, in silico analysis did not highlight any enrichment for canonical Hedgehog signaling (Fig. 4; Supplementary Fig. 4a-d). RT-qPCR further confirmed that the GLI-target genes, Gli1 and Ptch1, have the same level of expression in Gli3 $3^{+/+}$and Gli3 ${ }^{\Delta / \Delta}$ QSCs and ASCs (Supplementary Fig. 4e, f), suggesting that GLI3 does not repress its canonical target genes in satellite cells. Instead, Gene Ontology (GO) analysis of the 41 upregulated transcripts in Gli3 ${ }^{\mathrm{N} \Delta}$ QSCs revealed an enrichment for biological process terms related to glucose and insulin signaling (Fig. 4a; Supplementary Data file 2). In Gli3 ${ }^{\Delta / \Delta}$ ASCs, 144 transcripts are significantly upregulated and correlate with GO terms associated with regulation of G1/S phase transition and cell cycle (Fig. 4b; Supplementary Data file 2). The gene set enrichment analysis (GSEA) also confirmed the activation of cell cycle-related pathways (MYC/E2F targets, G2M checkpoint, DNA repair) in ASCs (Fig. 4d). Interestingly, mTORC1 signaling was identified among the active pathways in both Gli3 ${ }^{\Delta / \Delta}$ QSCs and ASCs (Fig. 4c, d). mTORC1 signaling activity drives the G0to- $G_{\text {Alert }}$ transition in quiescence ${ }^{6}$ and is required for satellite cell proliferation and fusion upon activation $^{38}$. Therefore, we hypothesized that Gli3 ${ }^{\Delta \Delta}$ satellite cells have transitioned into $\mathrm{G}_{\text {Alert }}$.

\section{GLI3R regulates the entry of quiescent satellite cells into $G_{\text {Alert }}$}

In contrast to quiescent $\mathrm{G}_{0}$ satellite cells, $\mathrm{G}_{\text {Alert }}$ satellite cells display an increase in cell size, transcriptional activity and mitochondrial metabolism, and are poised to activate faster in response to injury ${ }^{6}$. Imaging flow cytometry revealed that Gli3 $^{\Delta / \Delta}$ QSCs display an increase in size compared to Gli3 ${ }^{++}$QSCs (Fig. 5a-c; Supplementary Fig. 5a). Gli3 ${ }^{\Delta / \Delta}$ QSCs have increased PyroninY staining, consistent with increased transcriptional activity, and higher mitochondrial mass, in line with our RNA-sequencing data showing an enrichment in genes related to oxidative phosphorylation (Fig. 5d-g; Supplementary Fig. 5b). None of these features, however, overlaps 
with the profiles observed in ASCs, confirming that the Gli3 ${ }^{\Delta / \Delta}$ QSCs are not fully activated (Fig. 5b-g).

To assess their ability to enter the cell cycle, Gli3 ${ }^{+/+}$and Gli3 ${ }^{\Delta / \Delta}$ QSCs were cultured immediately after FACS-isolation in the presence of EdU for 40h (Fig. 5h). The proportion of EdU ${ }^{+}$

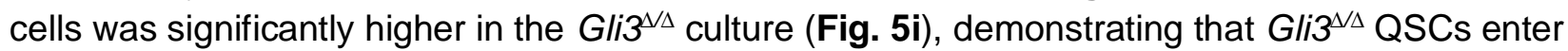
the cell cycle faster. This was further confirmed using freshly isolated myofibers cultured with EdU

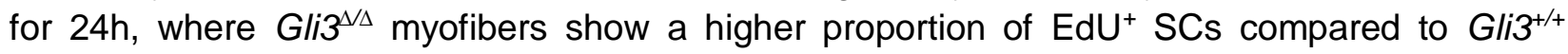
(Supplementary Fig. 5c-e).

As mTORC1 signaling regulates the $\mathrm{G}_{0}$-to- $\mathrm{G}_{\text {Alert }}$ transition in muscle stem cells ${ }^{6}$, we analyzed the phosphorylation of the ribosomal protein $S 6$ as a marker for mTORC1 activation in

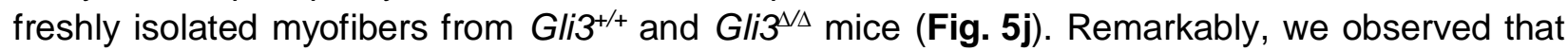
more than $50 \%$ of the Gli3 ${ }^{\Delta / \Delta}$ SCs display staining for phospho-S6, while only $20 \%$ of Gli3 $^{+/+}$SCs are phospho-S6+ (Fig. 5k). Altogether, these findings demonstrate that GLI3R represses mTORC1 signaling to maintain satellite cells in $G_{0}$ quiescence and that loss of GLI3R induces a transition to $\mathrm{G}_{\text {Alert. }}$

It has previously been demonstrated that $G_{0}$ and $G_{\text {Alert }}$ satellite cells share similar engraftment efficiency and capacity for self-renewal ${ }^{6}$. To confirm that $G\left(3^{\Delta / \Delta}\right.$ satellite cells also retain stemness properties, we performed transplantation experiments of $\mathrm{Gli}^{+/+}$and $\mathrm{Gli} \mathrm{3}^{\mathrm{L} \Delta}$ QSCs into irradiated TA muscles of immunocompromised MDX mice, a mouse model for Duchenne muscular dystrophy (Supplementary Fig. 5f). Remarkably, Gli3 ${ }^{\mathrm{V} \Delta} \mathrm{SCs}$ exhibit enhanced capacity to engraft and self-renew as they generate 3.5 times more $\mathrm{DYS}^{+} / \mathrm{YFP}^{+}$myofibers and PAX7 ${ }^{+/} \mathrm{YFP}^{+}$ satellite cells than the Gli3 ${ }^{+/+}$SCs (Supplementary Fig. 5g-j). Given that enhanced engraftment is not a phenotype associated with $\mathrm{G}_{\text {Alert }}$ satellite cells, this indicates that Gli3 deletion must affect other aspects of satellite cell biology beyond cell cycle regulation and, specifically, may affect satellite cell self-renewal.

\section{GLI3R regulates the self-renewal of satellite cells}

To evaluate the role of GLI3 in regulating satellite cell self-renewal, we utilized the $M y f 5^{\text {cre }} ; R 26 R^{\text {YFP }}$ mouse model. These mice allow for the tracking of the expression of the myogenic determination factor Myf5, following the first division of YFP- stem cells that have never expressed $M^{-} f^{7}$. Therefore, they allow analysis of asymmetric divisions and the early commitment of satellite cells ${ }^{7,39,40}$.

To assess the effects of GLI3 depletion or loss of GLI3 processing, we treated single EDL $M y f 5^{\text {cre }} ; R 26 R^{Y F P}$ myofibers with either Gli3 or Ift88 siRNA, respectively (Fig. 6a). We examined satellite cells immediately after their first cell division at $42 \mathrm{~h}$ of culture, when the number of asymmetric and symmetric cell doublets reflects either the commitment or the expansion of the stem cell pool, respectively (Fig. 6b). Both Ift88 and Gli3 siRNA significantly decrease the number of asymmetric cell doublets (Fig. 6c, g). Although Gli3 siRNA does not significantly change the number of symmetric divisions of YFP' satellite cells (Fig. $\mathbf{6 d}, \mathbf{h}$ ), both siRNA treatments increase

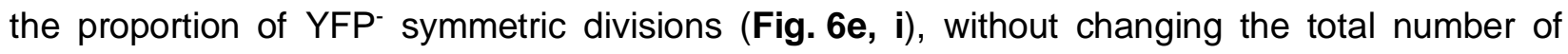
satellite cells per fiber (Fig. 6f, j). These data suggest that loss of GLI3 or disruption of its processing through ciliogenesis impairment drive satellite cell first division towards self-renewal and expansion rather than myogenic commitment. 
To test the ability of satellite cells to proliferate following their first division, single EDL myofibers from $\mathrm{Gli3}^{+/+}$and Gli3 ${ }^{\mathrm{N} \Delta}$ mice were cultured for $47 \mathrm{~h}$ and incubated with 5-ethynyl-2'deoxyuridine (EdU) $1 \mathrm{~h}$ before fixation (Supplementary Fig. 6a). At 48h, Gli3 ${ }^{\Delta / \Delta}$ satellite cells exhibit increased EdU incorporation along with an increase in the number of satellite cells per myofiber (Fig. 7a, b; Supplementary Fig. 6b). Analyzing in vivo EdU incorporation at 3 days postinjury further confirmed the enhanced ability of Gli3 ${ }^{\Delta / \Delta}$ ASCs to proliferate (Fig. 7c, d; Supplementary Fig. 6b). Single EDL myofibers from $\mathrm{Gli}^{+/+}$and $\mathrm{Gli} 3^{\mathrm{L} \Delta}$ mice were also cultured for $72 \mathrm{~h}$ to assess their differentiation potential. At this time point, the proportion of cells expressing the myogenic differentiation factor MYOGENIN (MYOG+) was significantly decreased upon Gli3 deletion (Fig. 7e, f), consistent with a reduction in generation of differentiation-competent progenitors due to decreased levels of asymmetric division ${ }^{41}$.

To determine whether Gli3 deletion permanently impairs or transiently delays myogenic differentiation, we derived primary myoblasts from $\mathrm{Gli3}^{+/+}$and $\mathrm{Gli}^{\mathrm{S} / \Delta}$ satellite cells in vitro (Supplementary Fig. 6a). Conversely to QSCs and ASCs, RT-qPCR analysis revealed that Gli3 $^{\Delta / \Delta}$ primary myoblasts display increased expression of Gli1 and Ptch1 (Supplementary Fig. 6c), suggesting that GLI3R regulates the expression of its canonical target genes in myogenic progenitors. This is also consistent with our results showing that in wild-type primary myoblasts, the modulation of GLI3R level directly affects Gli1 and Ptch1 expression. (Supplementary Fig. 2). However, in Gli3 ${ }^{\Delta / \Delta}$ myoblasts, SAG and FSK treatments failed to change the two GLI-target gene expression, excluding a role of GLI1 and GLI2 to compensate for the absence of GLI3 (Supplementary Fig. 6d, e). Gli3 ${ }^{\Delta / \Delta}$ myoblasts exhibit decreased expression of the myogenic regulatory factors, MYOD1, MYOGENIN, and the Myosin Heavy Chain (MyHC), at the early steps of differentiation (0-24h), yet they express similar levels of $\mathrm{MyHC}$ at the later steps (48-72h) and even form bigger myotubes than Gli3 ${ }^{+/}$cells (Fig. 7g; Supplementary Fig. 6c, f). This suggests that Gli3 ${ }^{\Delta / \Delta}$ myoblast differentiation is delayed rather than impaired. Together, these results show that Gli3 deletion increases satellite cell proliferation at the expense of early differentiation and delays, but does not prevent, terminal differentiation and fusion of myogenic progenitors.

\section{GLI3R controls the regenerative potential of muscle stem cells}

To assess their regenerative potential, Gli3 ${ }^{+/+}$and Gli3 $^{\Delta / \Delta}$ mice were subjected to a CTX-induced injury in the tibialis anterior (TA) muscle (Fig. 8a). At 7 days post-injury (d.p.i.), we counted the numbers of self-renewing and differentiating cells expressing either PAX7 or MYOG, respectively. An increased number of PAX7 $7^{+}$SCs was observed in Gli3 ${ }^{\Delta / \Delta}$ mice (Fig. 8b, c; Supplementary Fig. 7a), in accordance with increased satellite cell self-renewal (Fig. 6) and proliferation (Fig. 7ad). The number of $\mathrm{MYOG}^{+}$cells was slightly decreased compared to $\mathrm{Gli3}^{+/+}$mice (Supplementary Fig. 7b-c'), correlating with our ex vivo and in vitro findings suggesting a delay in differentiation (Fig. 7e-g). Of note, the morphology of the nascent myofibers expressing DYSTROPHIN (DYS) is improved in Gli3 ${ }^{\Delta / \Delta}$ mice, where interstitial space is reduced and myofibers are larger, suggesting that the delay in myogenic commitment does not impact the overall efficiency of regeneration (Fig. 8b; Supplementary Fig. 7b).

The number of PAX7 $7^{+}$SCs was increased in Gli3 ${ }^{\mathrm{N} \Delta}$ mice at 21 d.p.i., further suggesting enhanced self-renewal and expansion of the SC pool (Fig. 8d, e). Additionally, there was a significant increase in muscle mass mainly due to myofiber hypertrophy (Fig. 8f, g; Supplementary Fig. 7f-i') and increased numbers of centrally-located nuclei (Fig. 8h). This 
increase in myonuclear accretion suggests that, although there is a delay in terminal differentiation, the initial expansion of the satellite cell pool ultimately leads to a higher number of myogenic progenitors that eventually fuse into nascent and regenerating myofibers. Finally, in situ measurements of muscle force revealed that Gli3 ${ }^{\Delta / \Delta}$ regenerated TA muscles were $~ 50 \%$ stronger than Gli3 ${ }^{+/+}$muscles (Fig. 8i; Supplementary Fig. 7j). Together our data show that Gli3 deletion in satellite cells accelerates and improves muscle regeneration after CTX-induced trauma.

We also assessed the long-term regenerative capacity of the Gli3 ${ }^{\Delta / \Delta}$ satellite cells by challenging the mice with repetitive muscle injuries (Fig. 8j). Strikingly, Gli3 ${ }^{\Delta / \Delta}$ regenerated muscles are bigger, though some regions exhibit clear histological defects with fibrotic areas and tiny myofibers (Supplementary Fig. 7k). Even after three rounds of injury, Gli3 ${ }^{\mathrm{V} \Delta}$ mice maintain a higher number of self-renewing SCs (Fig. 8k, I), although their distribution throughout muscle section remains similar (Supplementary Fig. $7 \mathrm{~m}$ ). As observed following a single injury, Gli3 ${ }^{\Delta / \Delta}$ mice exhibited increased muscle weight resulting from both myofiber hyperplasia and hypertrophy (Fig. 8m; Supplementary Fig. 7I, n-p), and regenerating myofibers contained a higher number of centrally located nuclei (Supplementary Fig. 7q).

This again suggests that during each regeneration cycle, the stem cell pool expansion provides increasing number of myogenic progenitors that ultimately fuse to restore the damaged myofiber. Altogether, our data demonstrate that GLI3R regulates the expansion of the SC pool during muscle regeneration, and controls their regenerative potential.

\section{DISCUSSION}

Quiescent satellite cells lying in a non-cycling, dormant state have a primary cilium. Our study, as well as others ${ }^{12,28,42}$, demonstrates that muscle cells are transiently and dynamically ciliated as they progress through the myogenic lineage, and that the primary cilium reassembles in selfrenewing stem cells. Here, we specifically identify the primary cilia-mediated processing of GLI3 as a downstream effector of the quiescent state, revealing a novel mechanism of muscle stem cell regulation. Indeed, we find that satellite cell-specific depletion of GLI3 induces Glert $_{\text {in }}$ QSCs. Moreover, we show that Gli3 deletion promotes stem cell expansion and enhances regenerative and engraftment potential, providing a proof-of-principle for prospective therapeutic applications of our findings.

Our results show that GLI3R colocalizes with PKA at the basal bodies of QSCs. Many studies have delineated the signaling networks that maintain satellite cell quiescence ${ }^{2,4,5,43-48}$. One such pathway, the NOTCH-COLV-Calcitonin receptor (CALCR) cascade, acts through the PKA pathway, which also promotes GLI3 phosphorylation and processing into the repressor form ${ }^{4,46,48}$. Therefore, one can hypothesize that CALCR-dependent maintenance of $\mathrm{G}_{0}$ in QSCs is mediated through GLI3 phosphorylation. Upon muscle damage, disruption of the stem cell niche downregulates NOTCH signaling and cAMP-PKA activity ${ }^{4,46}$, potentially abrogating GLI3 phosphorylation and processing, which could then promote satellite cell activation independent of Hedgehog ligand-receptor binding. It is highly likely that interaction between signaling networks, such as this, underlies the fine-tuned control of satellite cell activation during regeneration, and further investigations into such interplay will be essential for the development of our understanding of satellite cell biology.

While our study shows that GLI3 processing is required to maintain a dormant quiescent state, Gli3 deletion does not lead to autonomous activation in satellite cells but instead induces 
an "alert" quiescence. The $\mathrm{G}_{\text {Alert }}$ state was first described in quiescent stem cells subjected to systemic exposure of HGFA released from a distant muscle injury ${ }^{6,9}$. Interestingly, fully reduced HMGB1 released from a bone injury induces $\mathrm{G}_{\text {Alert }}$ in $\mathrm{SCs}$ through CXCR4 signaling ${ }^{10}$, suggesting that several signaling pathways can induce the $\mathrm{G}_{\text {Alert }}$ state in stem cells. In addition, Der Vartanian et al. demonstrated that a subpopulation of satellite cells is protected from dioxin pollutant through a cell intrinsic, mTORC1-dependent $\mathrm{G}_{\text {Alert }}$ response ${ }^{11}$, demonstrating that factors released upon injury are not mandatory to induce $G_{\text {Alert }}$. Here, we find that the intrinsic loss of GLI3R is sufficient to induce $\mathrm{G}_{\text {Alert }}$ in satellite cells in the absence of any systemic or extrinsic cues. In wild-type condition, processing of GLI3 is likely to be responsive to both extrinsic and intrinsic cues, such as the CALCR discussed above. Hence, we propose that cilia-mediated GLI3 processing controls muscle stem cell quiescence and regulates the first step of entering $G_{\text {Alert }}$ in a physiological context.

Gli3 $^{\Delta / \Delta}$ resting muscle exhibit a slight increase in the number of satellite cells two weeks post-tamoxifen treatment. However, this number remains stable over time, and our results indicate that Gli $3^{\Delta / \Delta}$ satellite cells return to and maintain quiescence under homeostatic conditions. Satellite cells contribute to uninjured myofiber homeostasis in adulthood ${ }^{49,50}$. Since Gli3 deletion leads to enhanced cell proliferation and self-renewal, we speculate that the initial depletion of Gli3 triggers a transient entry into the cell cycle with a preference for self-renewal, increasing the total number of satellite cells before homeostatic quiescence is re-asserted.

Intriguingly, our RNA-sequencing data indicates that deleting Gli3 from the QSCs and ASCs does not significantly impact the expression of the putative GLI-target genes of the canonical Hedgehog pathway, notably Gli1 or Ptch1. Instead, loss of GLI3R induces the activation of mTORC1 signaling. Interestingly, the upregulation of the GLI transcription factors in ischemia/reperfusion injury protects muscle tissue through activation of AKT/mTOR/p70S6K signaling ${ }^{51}$. Several studies have highlighted the existence of a GLI-mediated mTORC1 activation $^{52,53}$, where GLI transcription factors regulate positively mTORC1 signaling by downregulation of negative or upregulation of positive mTORC1 mediators ${ }^{54}$. Both Gli3 $^{+/+}$and Gli3 ${ }^{\Delta / \Delta}$ QSCs and ASCs display decreased expression of Deptor, a negative regulator of mTORC1. Although Deptor is not a direct target of GLI3, downregulation of Deptor in Gli3 $3^{\Delta / \Delta}$ satellite cells is consistent with increased mTORC1 activity and S6 phosphorylation, consequently leading to $\mathrm{G}_{\text {Alert }}$ transition in Gli3 ${ }^{\Delta / \Delta}$ QSCs as well as enhanced proliferation in $\mathrm{Gli}^{\Delta / \Delta} \mathrm{ASCs}^{6,38}$.

Activated satellite cells undergo either asymmetric or symmetric division, giving rise to one committed muscle progenitor and one self-renewing stem cells, or to two self-renewing stem cells, respectively 7,55 . Stimulating symmetric stem cell expansion is associated with improved muscle regeneration ${ }^{39,40,56}$. Interestingly, GSEA revealed that Gli3 ${ }^{\Delta / \Delta}$ satellite cells display inhibition of the JAK/STAT signaling, which knockdown or pharmacological inhibition has been shown to favor satellite cell expansion, homing and regenerative capacity, overall improving skeletal muscle repair ${ }^{40,56}$. Thus, the decreased activity of JAK/STAT signaling in Gli3 ${ }^{\mathrm{N} \Delta}$ satellite cells is consistent with their enhanced ability to self-renew and regenerate. As well, GO analysis on the 629 downregulated genes highlighted biological process terms mainly related to cell adhesion, supporting the idea that Gli3 ${ }^{\Delta \Delta}$ ASCs are less adhesive to their environment during regeneration, allowing more efficient migration and muscle tissue repair ${ }^{2,57}$. Corroborating this result, GSEA revealed the inhibition of cell-cell interaction mechanisms (Apical junction/surface, Epithelial- 
mesenchymal transition). Thus, increased self-renewal, decreased adhesion and enhanced proliferation overall improves the regenerative capacity of Gli3 ${ }^{\mathrm{N} \Delta}$ 'alert' satellite cells.

Nevertheless, sustained cell proliferation can compromise myogenic differentiation and impair muscle regeneration ${ }^{56}$. As previously shown ${ }^{58}$, the absence of GLI3 in myogenic progenitors increases their proliferation at the expense of early differentiation. However, while Gli3 deletion in differentiated muscle cells delays the overall muscle regeneration ${ }^{58}$, Gli3 deletion in satellite cells leads to opposite. Gli3 ${ }^{\Delta / \Delta}$ satellite cells form larger myotubes upon differentiation in vitro and promote myofiber hypertrophy in vivo. Likely, this discrepancy is the result of the expansion of satellite cells at the early stages of regeneration/myogenesis, providing a larger pool of progenitor myoblasts that eventually, after a delay, fuse into myofibers or myotubes. The expansion is mediated both by the $\mathrm{G}_{\text {Alert }}$ state, conferring accelerated proliferative properties to satellite cells, and the increased propensity for self-renewal divisions. Ultimately, the initial expansion compensates for the delay in myogenic commitment, leading to better repair in Gli3 $3^{\Delta / \Delta}$ mice. As we and others showed ${ }^{42}$, GLI3 is down-regulated during the later stages of myogenic differentiation, excluding a potential role for GLI3 after satellite cell commitment. This suggests that other signals emanating from the regenerating muscle are sufficient to induce myogenesis ${ }^{59}$ and this response does not appear to be impaired in Gli3 ${ }^{\Delta / \Delta}$ satellite cells.

Different studies have explored the beneficial effects of activating canonical Hedgehog signaling to induce myogenic progenitor proliferation and differentiation during muscle repair ${ }^{51,60-}$ 65. Our results show that satellite cells do not activate canonical Hedgehog signaling within the first 3 days following an acute injury. While deleting Gli3 from satellite cells does not activate the expression of the canonical GLI-target genes, increased expression of Gli1 and Ptch1 was observed in SAG-treated myoblasts and Gli3 ${ }^{\Delta / \Delta}$ myogenic progenitors, suggesting that they can respond to Hedgehog ligands. At day 5 following CTX-induced acute injury, when the population of myogenic progenitors is predominant, $\mathrm{DHH}$ is robustly expressed by the Schwann cells ${ }^{27}$. Thus, the induction of $\mathrm{DHH}$ might contribute to the proliferation and differentiation of the myogenic progenitors. Our results imply that GLI3R has pleiotropic roles during muscle stem cell progression through the myogenic lineage: controlling satellite cell quiescence and activation independently of its canonical target genes while repressing Hedgehog signaling target genes in myogenic progenitors to regulate their proliferation and differentiation.

Our results demonstrate that the primary cilium-mediated control of GLI3 processing regulates muscle stem cell fate and that loss of GLI3R from activated satellite cells and myoblasts results in increased proliferation and self-renewal. However, studies regarding the ablation of primary cilia from muscle cells have led to different and somewhat conflicting results ${ }^{12,42}$. In the C2C12 myogenic cell line, Ift88 knockdown results in increased proliferation at the expense of differentiation ${ }^{42}$. Conversely, drug-mediated cilia disassembly does not affect satellite cell proliferation and differentiation but impairs self-renewal ${ }^{12}$. This discrepancy is likely related to the experimental settings and differences in the behavior of myogenic progenitors and satellite cells, as we showed. As well, these data suggest that the primary cilium has broader signaling function in muscle cells than the only regulation of GLI3 proteolytic processing.

Although we largely observed beneficial effects of GLI3 depletion, we cannot exclude the possibility that long-term loss of GLI3 repressor function can have negative consequences, as observed in other knockout models ${ }^{66}$. Gli3 ${ }^{\Delta / \Delta}$ regenerated muscles following a triple injury exhibit signs of histological defects, suggesting that permanent GLI3R depletion may eventually lead to 
regenerative deficits over time. Along these lines, given the importance of quiescence regulation in preventing precocious activation and maintaining the satellite cell pool over an organism's lifetime, one could speculate that the negative consequences of $G / 3^{\Delta / \Delta}$ would not be observed until much later ages. As well, the persistence of a $\mathrm{G}_{\text {Alert }}$ state in uninjured homeostasis represents an inefficient use of energetic and substrate resources within muscle, which could be detrimental in contexts of metabolic scarcity. Therefore, translational studies should aim to determine the benefits of transient GLI3 depletion or the pharmacological inhibition of its proteolytic cleavage for the development of stem cell-based therapeutic strategies in regenerative medicine ${ }^{44,66-68}$.

Our findings represent a seminal advancement in our understanding of the molecular regulation of adult muscle stem cell function. This study establishes, for the first time, that primary cilia-mediated GLI3 processing controls the transition from $\mathrm{G}_{0}$-to- $\mathrm{G}_{\text {Alert }}$, as well as the fatedeterminate division that follows satellite cell activation. As a result, GLI3 processing within the primary cilia directly impacts the regenerative capacity of stem cells. Further studies are needed to understand how endogenous signaling events or mechanosensing by the primary cilia can control GLI3 processing in response to injury. Also, the downstream signaling events that mediate GLI3R activity remain to be determined. Finally, pharmacological methods of manipulating GLI3 processing warrant investigation for their potential use in muscle stem cell-based therapies. 


\section{METHODS}

\section{Mouse strains and animal care}

The following mouse lines were used in this study: Gli3fl/fl mice69, Pax7CreERT2/+ mice70 referred as $P a x 7^{C E /}$ in the text, Myf5 ${ }^{C r e}$ mice $^{71}, R 26 R^{E Y F P}$ mice ${ }^{72}$ referred as $R 26 R^{Y F P}$, Pax7nGFP mice $^{73}$, C57BL/10ScSn-Dmd ${ }^{m d x} / J$ mice (homozygous $D m d^{m d x}$ females and hemizygous $D m d^{m d x}$ males) referred as MDX in the manuscript. All the mice used in this study were males and females, from 2 to 10 month-old, with mixed genetic background (129SV and C57BL/6). Mice were sex and age-matched in all experiments. Housing, husbandry and all experimental protocols for mice used in this study were performed in accordance with the guidelines established by the University of Ottawa Animal Care Committee, which is based on the guidelines of the Canadian Council on Animal Care (CCAC). Protocols were approved by Animal Research Ethics Board (AREB) at the University of Ottawa.

\section{Tamoxifen treatment and muscle injury}

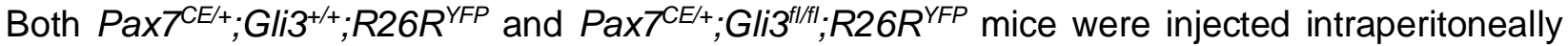
with $100 \mu \mathrm{l}$ of a $20 \mathrm{mg} \cdot \mathrm{ml}^{-1}$ tamoxifen solution (TMX, Sigma T5648) dissolved in corn oil for 4 consecutive days, and then they were maintained on a diet containing tamoxifen (500mg TMX per kg diet, Teklad, Envigo). Muscle injury was induced by intramuscular injections of cardiotoxin

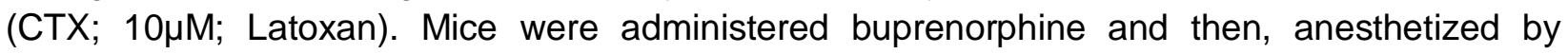
isofluorane inhalation. For histological analysis, $50 \mu \mathrm{l}$ of was injected into tibialis anterior (TA) muscle. For flow cytometry and FACS analysis, both TA and gastrocnemius muscles were injected with $40 \mu \mathrm{l}$ and $80 \mu \mathrm{l}$ of CTX, respectively.

\section{Muscle fixation and histological analysis}

Mice were euthanized and TA muscles were harvested, weighed and embedded in OCT and frozen in liquid nitrogen-cooled isopentane. For GFP visualization, mice were euthanized with a sodium pentobarbital solution (Euthanyl) and perfused with PBS before fixation with $4 \%$ paraformaldehyde (PFA) solution in PBS. Muscles were excised, further fixed into $1 \%$ PFA/PBS for $16 \mathrm{~h}$, then transferred into ascending sucrose gradients (15\%,30\% in PBS), and finally embedded and frozen in OCT. Embedded muscles were transversely sectioned at $10 \mu \mathrm{m}$ thickness. Sections were post-fixed in 4\% PFA/PBS $10 \mathrm{~min}$ at room temperature, permeabilized in $0.1 \mathrm{M}$ glycine, $0.1 \%$ Triton X-100 in PBS, and blocked in 5\% goat serum, $2 \%$ BSA in PBS supplemented with M.O.M. Blocking reagent (Vector Laboratories). Then, sections were incubated with primary antibodies as described in "Immunostaining on cells, myofibers and sections". Muscle cross-sections stained with anti-Laminin and anti-Dystrophin and counterstained with DAPI were analyzed for fiber counting and minimum Feret's diameter using $\mathrm{SMASH}^{74}$, and centronuclei per myofiber were quantified using MuscleJ ${ }^{75}$.

\section{Flow cytometry and Fluorescence-activated cell sorting (FACS)}

Quiescent satellite cells were obtained from uninjured hindlimb muscles, while activated satellite cells were obtained from CTX-injured tibialis anterior and gastrocnemius muscles 3 days after the induced-injury. Dissected muscles were minced in collagenase/dispase solution followed by dissociation using the gentleMACS Octo Dissociator with Heaters (Miltenyi Biotec). Satellite cells were sorted by gating a mononuclear cell population of $\alpha 7-I N T E G R I N^{+}, C D 34^{+}, C D 31^{\text {neg }}, C D 45^{\text {neg }}$, 
SCA1 ${ }^{\text {neg }}, C D 11 b^{\text {neg }}$ (quiescent satellite cells) or a7-INTEGRIN ${ }^{+}, V_{C A M} 1^{+}, C D 31^{\text {neg }}, C 45^{\text {neg }}$, SCA1 ${ }^{\text {neg }}, C D 11 b^{\text {neg }}$ (activated satellite cells) using a MoFlo XDP cell sorter (Beckman Coulter). Gating strategy is shown in Supplementary Fig. 8. Flow cytometry analyses (PyroninY, MitoTracker) were performed on a BD LSRFortessa cell analyzer (BD Biosciences). The list of antibodies is available in Supplementary Table 1.

\section{Cell size measurement}

Quiescent and activated satellite cells were sorted based for $\alpha 7-I N T E G R I N^{+}$, VCAM1 ${ }^{+}, C D 31^{\text {neg }}$, $\mathrm{CD} 45^{\text {neg }}, \mathrm{SCA} 1^{\text {neg }}, \mathrm{CD} 11 \mathrm{~b}^{\text {neg }}$. Single cell brightfield images were captured on the Amnis ImageStream XMk II and analyzed on the IDEAS Software.

\section{PyroninY and MitoTracker staining}

40nM PyroninY (Santa Cruz) and 40nM MitoTracker Deep Red (ThermoFisher) were added to the muscle digests and incubated $30 \mathrm{~min}$ at $37^{\circ} \mathrm{C}$ in a water bath. Then, muscle digests were washed and stained with the antibodies for gating the satellite cell population ( $\alpha 7$-INTEGRIN ${ }^{+}, \mathrm{VCAM}^{+}$, CD31 ${ }^{\text {neg }}, C D 45^{\text {neg }}, S C A 1^{\text {neg }}, C D 11 b^{\text {neg }}$ ). Gating strategy is shown in Supplementary Fig. $\mathbf{5 b .}$

\section{In vivo EdU incorporation assay}

Both tamoxifen-treated $P a x 7^{C E /+} ; G l i 3^{+/+} ; R 26 R^{Y F P}$ and $P a x 7^{C E /+} ; G l i 3^{f / / f /} ; R 26 R^{Y F P}$ mice were subjected to CTX-induced muscle injury in both TA and GA muscles. 3 days post-injury, mice were injected intraperitoneally with $10 \mu \mathrm{l}$ per gram of body weight of a $10 \mathrm{mM}$ EdU solution 3 hours before sacrifice. Then, muscles were collected and digested as described in 'Flow cytometry and Fluorescence-activated cell sorting' section.

\section{Satellite cell transplantation and engraftment assay}

Host MDX mice were anesthetized with isofluorane and hindlimbs were irradiated with 16Gy Xrays delivered at $0.71 \mathrm{~Gy} / \mathrm{min}$ with an X-RAD 320 (Precision X-Ray) biological irradiator. The following day, irradiated MDX mice were implanted subcutaneously with osmotic pumps (Alzet) delivering FK-506 immunosuppressant (LC Laboratories) at $2.5 \mathrm{mg} / \mathrm{kg} /$ day. Two days after, donor satellite cells from tamoxifen-treated $P a x 7^{C E /+} ; G / i 3^{+/+} ; R 26 R^{Y F P}$ and $P a x 7^{C E /+} ; G l i 3^{\text {fl/fl }} ; R 26 R^{Y F P}$ mice were FACS-isolated based on FSC/SSC, lineage negative selection (CD31, CD11b, CD45, SCA1), and positive selection ( $\alpha 7-$ INTEGRIN, CD34). Donor satellite cells were washed with PBS and resuspended in $0.9 \% \mathrm{NaCl}$ solution at a concentration of $10^{5}$ cells per $10 \mu$ prior to engraftment into the TA muscle of irradiated and immunosuppressed MDX mice. Transplanted TA muscles were collected two weeks after transplantation for measurement of satellite cell engraftment.

\section{EDL myofiber isolation, siRNA transfection and EdU treatment}

Myofibers were isolated from extensor digitorum longus (EDL) muscles following the previously described protocol ${ }^{76}$. Briefly, EDL muscles were dissected from tendon-to-tendon and incubated for $1 \mathrm{~h}$ in DMEM (Gibco) containing $0.25 \%$ collagenase I (Worthington). Single EDL myofibers were isolated by gentle muscle trituration and washed in DMEM. EDL myofibers were finally cultured in DMEM supplemented with $20 \%$ fetal bovine serum, $1 \%$ chick embryo extract and $1 \%$ penicillin/streptomycin. Myofibers were fixed at the desired time points using either PFA4\%/PBS or ice-cold methanol for immunostaining analysis. 
Single EDL myofibers were transfected with Gli3 siRNA (TriFECTa DsiRNA Kit mouse Gli3, mm.Ri.Gli3.13), Ift88 siRNA (TriFECTa DsiRNA Kit mouse Ift88, mm.Ri.Ift88.13) or scramble negative control siRNA, at a final concentration of $5 \mathrm{nM}$ using Lipofectamine RNAiMAX (Invitrogen) according to the manufacturer's instructions. siRNA transfection was performed twice at $4 \mathrm{~h}$ and $16 \mathrm{~h}$ post-culture and $6 \mathrm{~h}$ after the second transfection, growth medium was renewed.

For EdU treatment, freshly isolated EDL myofibers were treated for $24 \mathrm{~h}$ with $20 \mu \mathrm{M}$ EdU (ThermoFisher) to analyze satellite cell activation. For cell proliferation analysis, 48h-cultured myofibers were treated with $20 \mu \mathrm{M}$ EdU for $1 \mathrm{~h}$ before fixation.

\section{Myoblast isolation, culture and treatments}

8-16 week-old mice were used to derive primary myoblasts by magnetic cell separation (MACS) ${ }^{77}$. Muscle dissociation and cell filtration was performed following the same protocol described for Flow cytometry and FACS. First, negative lineage selection was performed with biotin-conjugated lineage antibodies (CD11b, SCA1, CD45, CD31), followed by incubation with streptavidin microbeads. Then, satellite cell-derived myoblasts were purified using biotin-conjugated anti- $\alpha 7-$ INTEGRIN antibody. Myoblasts were cultured on collagen-coated dishes in Ham's F10 medium (Wisent) supplemented with $20 \%$ FBS, $1 \%$ penicillin/streptomycin, and $5 \mathrm{ng} \cdot \mathrm{ml}^{-1}$ of basic FGF (Millipore). Differentiation was induced in Ham's F10:DMEM 1:1 supplemented with 5\% horse serum, and $1 \%$ penicillin/streptomycin.

For GLI3 proteolytic processing analysis, primary myoblasts were treated with either 200mM SAG (Smoothened agonist, R\&D Systems) or 25 $\mu$ M FSK (Forskolin, R\&D Systems) for 24 hours. Equivalent amounts of DMSO were added to the control conditions.

\section{Reserve cell isolation}

Primary myoblasts were derived from 8-16 week-old Pax7-nGFP mice and cultured as described above. Myoblasts were differentiated for 3 days. Then, total cells were trypsinized, washed in PBS and resuspended in FACS buffer. 'Reserve' cells were sorted by gating a mononuclear GFP ${ }^{+}$cell population using the MoFlo XDP cell sorter (Beckman Coulter).

\section{RNA extraction and quantitative PCR}

Total RNA was extracted from primary myoblasts using the Nucleospin RNA II kit (MachereyNagel) and from satellite cells using the ARCTURUS Picopure RNA extraction kit (ThermoFisher), according to the manufacturers' instructions. Reverse transcription was performed using SuperScript III Reverse Transcriptase (Invitrogen). Gene expression was assessed with iQ SYBR Green Supermix (Bio-Rad) and analysis was performed using the $2^{-\Delta \Delta C t}$ method. RT-qPCR were normalized to the housekeeping genes Ppia. A list of primers is available in Supplementary Table 2.

\section{RNA-sequencing and gene expression analysis}

12 samples were used for RNA-sequencing analysis: 3 samples of quiescent satellite cells and 3

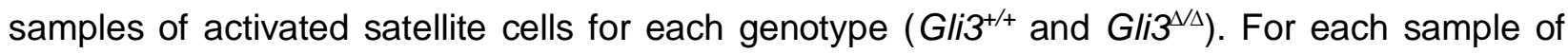
quiescent satellite cells, all hindlimb muscles from 2-3 mice were combined. Activated satellite cells were isolated from injured TA and gastrocnemius muscles at day 3 post-CTX injections, and each sample corresponded to one mouse. 
Library construction was performed with 20ng of input total RNA using the NEBNext Ultra II Directional RNA Library Prep Kit for Illumina - polyA mRNA workflow (New England Biolabs). The libraries were sequenced with a NextSeq 500 High Output 75 cycle kit (Illumina). RNA-seq reads were mapped to transcripts from GRCm38_GENCODE.vM19 using salmon $\mathrm{v} 0.13 .1^{78}$. Data were loaded into $\mathrm{R}$ using the tximport library and the gene/count matrix was filtered to retain only genes with five or more mapped reads in two or more samples. Differential expression was assessed using DESeq2 ${ }^{79}$. PCA was performed using the DESeq2 plotPCA function and rlog-transformed count data. Pearson correlation between means of CPM normalized expression for each replicate group was calculated. Expression differences were calculated using the IfcShrink function, applying the apeglm method (v1.6.0 $)^{80}$. Gene Ontology (GO) analysis and Gene set enrichment analysis (GSEA) were performed using goseq (v1.40.0) and fgsea (v1.14.0) $R$ packages, respectively, on the significantly upregulated and downregulated genes (cut-off of 0.05 and absolute fold changes greater than or equal to 1.2) from $\mathrm{Gli3}^{+/+}$and $\mathrm{Gli}^{\mathrm{\Delta} / \Delta}$ QSCs and ASCs.

\section{Western blotting}

Whole cell proteins were extracted in lysis buffer $(150 \mathrm{mM} \mathrm{NaCl}, 25 \mathrm{mM}$ Tris pH7.5, $1 \%$ NP-40, $0.5 \%$ sodium deoxycholate, $0.1 \%$ SDS) supplemented with inhibitors of proteases (Roche) and phosphatases (Sigma). Equal amounts of proteins were resolved on SDS-PAGE 4-12\% (Bio-Rad) and transferred onto PVDF membranes. Membranes were blocked using 5\% non-fat dry milk in TBS-Tween $0.1 \%$ (TBST) for $1 \mathrm{~h}$ at room and probed with primary antibodies overnight at $4^{\circ} \mathrm{C}$. The list of antibodies is available in Supplementary Table 1. After 4 washes in TBST, membranes were incubated $1 \mathrm{~h}$ with HRP-conjugated secondary antibodies at 1:5,000 (Bio-Rad). After 4 more washes, immunoblots were developed by enhanced chemiluminescence. When required, PVDF membranes were stripped in $62.5 \mathrm{mM}$ Tris $\mathrm{HCl}, \mathrm{pH} 6.8,2 \%$ SDS and $0.8 \% \beta$-mercaptoethanol.

\section{Immunostaining on cells, myofibers and sections}

Following fixation in PFA 2\%/PBS, cells and myofibers were washed 2 times in PBS, permeabilized in $0.1 \mathrm{M}$ Glycine, $0.1 \%$ Triton X-100 in PBS for $10 \mathrm{~min}$. For EdU staining, samples were stained using the Click-iT EdU Alexa Fluor 647 Imaging kit (ThermoFisher), according to the manufacturer's instructions. Then, cells and myofibers were blocked in $5 \%$ horse serum, $2 \%$ BSA, $0.1 \%$ Triton X-100 in PBS for at least $1 \mathrm{~h}$, and incubated with primary antibodies overnight at $4^{\circ} \mathrm{C}$. The list of antibodies is available in Supplementary Table 1. Samples were washed 3 times in PBS, incubated $1 \mathrm{~h}$ at room temperature with Alexa Fluor-conjugated secondary antibodies at 1:1,000 (ThermoFisher), washed 3 times in PBS and counterstained with DAPI at $1 \mu \mathrm{g} \cdot \mathrm{mL}^{-1}$ in PBS before mounting.

For GLI3/acaTUB/PAX7 co-staining, cells and myofibers were first incubated with antiGLI3 primary antibody and then, Alexa Fluor donkey anti-goat secondary antibody. Following 2 washes in PBS-Tween $0.1 \%$ and PBS, cells and myofibers were incubated with anti-acaTUB and anti-PAX7 primary antibodies followed by Alexa Fluor goat anti-mouse lgG2b and goat anti-mouse lgG1 secondary antibodies.

YTUB immunostaining required ice-cold methanol fixation. Briefly, cells and myofibers were rinsed two times in PBS, incubated $30 \mathrm{~min}$ in ice-cold $\mathrm{MeOH}$ at $-20^{\circ} \mathrm{C}$ and rehydrated in successive 
washes of $50 \% \mathrm{MeOH} / 50 \%$ PBS, $30 \% \mathrm{MeOH} / 70 \%$ PBS and PBS before being incubated in blocking buffer.

Full muscle section pictures were taken on a Zeiss Axio Observer.D1 inverted microscope equipped with an EC Plan-Neofluar 10×/0.3 Ph1 M27 objective and stitched together using Fiji software (http://fiji.sc/Fij). Other immunofluorescence pictures were taken with a Zeiss Axio Observer.D1 inverted microscope equipped with either a Plan-Apochromat 20×/0.8 M27 objective or a Plan-Apochromat 63×/1.4.Oil DIC M27 objective. For GLI3 localization, images were taken with a confocal Zeiss LSM 880 AiryScan inverted microscope quipped with a Plan-Apochromat $63 \times / 1.4$.Oil DIC M27 objective. Images were processed and analyzed with Zen and FIJI software.

\section{In situ force measurement}

Muscle force measurements were performed on an Aurora Scientific 300C-LR-FP dual mode muscle lever system equipped with a $1 \mathrm{~N}$ force transducer and $1 \mathrm{~cm}$ lever arm. Electrical stimulation was performed using monopolar needle electrodes attached to an Aurora Scientific 701C HighPower, Bi-Phase Stimulator. Force transducers were calibrated prior to the study using precision weights. Mice were anesthetized using isoflurane inhalation $(2 \%$ isoflurane, $1 \mathrm{~L} / \mathrm{min})$ until recumbent and non-reflexive to pressure on the paw and positioned on a heated pad to maintain their body temperature at $37^{\circ} \mathrm{C}$ throughout the procedure. Mice were positioned supine and hindlimb were shaved. A small incision was made above the hallux and the foot was partially degloved to expose the distal insertion of the tibialis anterior tendon up to the tibialis anterior muscle. The cruciate crural ligament was severed to release the tibialis anterior (TA) tendon from the foot.

A pre-tied loop of waxed 3.5 metric suture was attached to the TA tendon using a series of double thumb knots above, below and through the loop. The suture was secured to the tendon using minimal amounts of cyanoacrylate glue. The skin of the hindlimb was removed up to mid vastus lateralis to expose the TA and the kneecap. The fascia of the TA was cut using spring scissors. The distal insertion of the TA tendon was severed and the TA was gently lifted to release it from the extensor digitorum longus (EDL) muscle and connective tissue. Muscles were kept from drying using physiological saline. The measured hindlimb was secured between the limb clamp and the stage using a $40 \mathrm{~mm}$ long $27 \mathrm{~g}$ needle inserted through the epiphysis of the femur immediately proximal the kneecap and directly into a receiving hole in the stage. Clamping was verified by observing no movement of the kneecap following manipulation of the foot and the needle was secured by a hand screw. The pre-tied loop was attached to the hook on the force transducer lever arm and maintained without tension.

Two monopolar needle electrodes were positioned adjacent to the tibial nerve proximal to the kneecap and distal the kneecap adjacent the EDL muscle. The transducer was retracted to maintain $20 \mathrm{mN}$ of measured tension for an initial 15 minute stretching period with $100 \mathrm{~ms}$ trains of $0.3 \mathrm{~ms}, 5 \mathrm{~V}$ supramaximal voltage pulses at $1 \mathrm{~Hz}$ stimulation every 100 seconds. Following stretching, muscles were maintained at $20 \mathrm{mN}$ tension and tetanic contractions were measured every 100 seconds following $200 \mathrm{~ms}$ trains of $0.3 \mathrm{~ms}$, $5 \mathrm{~V}$ supramaximal voltage pulses at serial frequencies from $1 \mathrm{~Hz}$ to $200 \mathrm{~Hz}$. Maximal force was defined by the difference in maximal force measured during stimulation to that of the tension immediately prior stimulation. 


\section{Statistical analysis}

No statistical method was used to predetermine sample size. Statistical evaluation was performed using the Student's t-test tests to calculate differences between two groups and either one-way or two-way ANOVA with post hoc test for multiple comparisons (Graphpad Prism®), Data Source file). The number of independent experimental replications is reported in each corresponding figure legend. Data are presented as mean \pm S.E.M. and $p$-value $<0.05$ was considered as statistically significant. Throughout the manuscript, level of significance is indicated as follows: ${ }^{*} p$ $\leq 0.05,{ }^{* *} p \leq 0.01,{ }^{* * *} p \leq 0.001$.

\section{ACKNOWLEDGMENTS}

The authors thank Dr. Valerie Wallace for providing the Gli3 floxed mice, Jennifer Ritchie for animal husbandry, Fernando Ortiz for FACS, Caroline Vergette from StemCore Laboratories, Gareth Palidwor from Bioinformatics Core, Hani Jrade and Damian Carragher for helping with the ImageStream flow cytometry, Alireza Ghasemizadeh for helping with confocal imaging, Hong Ming, Ricardo Carmona and Pascale Muller for technical assistance and Sandy Martino for administrative assistance. C.E.B. was supported by postdoctoral fellowships from the Ontario Institute for Regenerative Medicine (OIRM) and the French Muscular Dystrophy Association (AFM)-Téléthon and is now supported by a postdoctoral fellowship from the Fondation pour la Recherche Médicale [FRM, ARF201909009155]. A.Y.T.L. is supported by a postdoctoral fellowship from OIRM. P.F. was supported by a doctoral fellowship from the Canadian Institutes of Health Research (CIHR). Studies from the F.L.G. lab were supported by grants from the Agence Nationale pour la Recherche (ANR): Myofuse project [ANR-19-CE13-0016-03] and Myofibrosis project [ANR-19-CE14-0008-02], and from the European Joint Programme on Rare Diseases (EJP RD): MYOCITY project. M.A.R. holds a Canada Research Chair in Molecular Genetics. These studies were carried out with the support of grants from the US National Institutes for Health [R01AR044031], the Canadian Institutes of Health Research [FDN-148387], and the Stem Cell Network.

\section{AUTHOR CONTRIBUTIONS}

C.E.B. and M.A.R. conceived the project. C.E.B., M.-C.S. and A.Y.T.L. designed methods. C.E.B., M.-C.S., A.Y.T.L. and D.H. conducted experiments and analyzed results. W.J. and F.L.G. performed in silico analysis of RNA-sequencing data. P.F. performed force measurement and analysis. M.R. performed satellite cell transplantation. C.E.B. wrote the original draft with input from co-authors. C.E.B. and M.A.R. edited the final manuscript. M.A.R. and F.L.G. provided financial support.

\section{COMPETING INTERESTS STATEMENT}

The authors declare no competing interests. 


\section{REFERENCES}

1 Dumont, N. A., Bentzinger, C. F., Sincennes, M. C. \& Rudnicki, M. A. Satellite Cells and Skeletal Muscle Regeneration. Comprehensive Physiology 5, 1027-1059, doi:10.1002/cphy.c140068 (2015).

2 Eliazer, S. et al. Wnt4 from the Niche Controls the Mechano-Properties and Quiescent State of Muscle Stem Cells. Cell stem cell, doi:10.1016/j.stem.2019.08.007 (2019).

3 Goel, A. J., Rieder, M. K., Arnold, H. H., Radice, G. L. \& Krauss, R. S. Niche Cadherins Control the Quiescence-to-Activation Transition in Muscle Stem Cells. Cell reports 21, 2236-2250, doi:10.1016/j.celrep.2017.10.102 (2017).

4 Baghdadi, M. B. et al. Reciprocal signalling by Notch-Collagen V-CALCR retains muscle stem cells in their niche. Nature 557, 714-718, doi:10.1038/s41586-018-0144-9 (2018).

5 Baghdadi, M. B. et al. Notch-Induced miR-708 Antagonizes Satellite Cell Migration and Maintains Quiescence. Cell stem cell 23, 859-868 e855, doi:10.1016/j.stem.2018.09.017 (2018).

6 Rodgers, J. T. et al. mTORC1 controls the adaptive transition of quiescent stem cells from G0 to G(Alert). Nature 510, 393-396, doi:10.1038/nature13255 (2014).

$7 \quad$ Kuang, S., Kuroda, K., Le Grand, F. \& Rudnicki, M. A. Asymmetric self-renewal and commitment of satellite stem cells in muscle. Cell 129, 999-1010, doi:10.1016/j.cell.2007.03.044 (2007).

8 Collins, C. A. et al. Stem cell function, self-renewal, and behavioral heterogeneity of cells from the adult muscle satellite cell niche. Cell 122, 289-301, doi:10.1016/j.cell.2005.05.010 (2005).

9 Rodgers, J. T., Schroeder, M. D., Ma, C. \& Rando, T. A. HGFA Is an Injury-Regulated Systemic Factor that Induces the Transition of Stem Cells into GAlert. Cell reports 19, 479-486, doi:10.1016/j.celrep.2017.03.066 (2017).

10 Lee, G. et al. Fully reduced HMGB1 accelerates the regeneration of multiple tissues by transitioning stem cells to GAlert. Proceedings of the National Academy of Sciences of the United States of America 115, E4463-E4472, doi:10.1073/pnas.1802893115 (2018).

11 Der Vartanian, A. et al. PAX3 Confers Functional Heterogeneity in Skeletal Muscle Stem Cell Responses to Environmental Stress. Cell stem cell 24, 958-973 e959, doi:10.1016/j.stem.2019.03.019 (2019).

12 Jaafar Marican, N. H., Cruz-Migoni, S. B. \& Borycki, A. G. Asymmetric Distribution of Primary Cilia Allocates Satellite Cells for Self-Renewal. Stem cell reports, doi:10.1016/j.stemcr.2016.04.004 (2016).

13 Goetz, S. C. \& Anderson, K. V. The primary cilium: a signalling centre during vertebrate development. Nature reviews. Genetics 11, 331-344, doi:10.1038/nrg2774 (2010).

14 Bangs, F. \& Anderson, K. V. Primary Cilia and Mammalian Hedgehog Signaling. Cold Spring Harbor perspectives in biology 9, doi:10.1101/cshperspect.a028175 (2017).

15 Anvarian, Z., Mykytyn, K., Mukhopadhyay, S., Pedersen, L. B. \& Christensen, S. T. Cellular signalling by primary cilia in development, organ function and disease. Nature reviews. Nephrology 15, 199-219, doi:10.1038/s41581-019-0116-9 (2019).

16 Haycraft, C. J. et al. Gli2 and Gli3 localize to cilia and require the intraflagellar transport protein polaris for processing and function. PLoS genetics 1, e53, doi:10.1371/journal.pgen.0010053 (2005). 
17 Huangfu, D. \& Anderson, K. V. Cilia and Hedgehog responsiveness in the mouse. Proceedings of the National Academy of Sciences of the United States of America 102, 1132511330, doi:10.1073/pnas.0505328102 (2005).

18 May, S. R. et al. Loss of the retrograde motor for IFT disrupts localization of Smo to cilia and prevents the expression of both activator and repressor functions of Gli. Developmental biology 287, 378-389, doi:10.1016/j.ydbio.2005.08.050 (2005).

$19 \mathrm{Li}$, J. et al. PKA-mediated Gli2 and Gli3 phosphorylation is inhibited by Hedgehog signaling in cilia and reduced in Talpid3 mutant. Developmental biology 429, 147-157, doi:10.1016/j.ydbio.2017.06.035 (2017).

20 Niewiadomski, P. et al. Gli protein activity is controlled by multisite phosphorylation in vertebrate Hedgehog signaling. Cell reports 6, 168-181, doi:10.1016/j.celrep.2013.12.003 (2014). 21 Wen, X. et al. Kinetics of hedgehog-dependent full-length Gli3 accumulation in primary cilia and subsequent degradation. Molecular and cellular biology 30, 1910-1922, doi:10.1128/MCB.01089-09 (2010).

22 Mick, D. U. et al. Proteomics of Primary Cilia by Proximity Labeling. Developmental cell 35, 497-512, doi:10.1016/j.devcel.2015.10.015 (2015).

23 Tuson, M., He, M. \& Anderson, K. V. Protein kinase A acts at the basal body of the primary cilium to prevent Gli2 activation and ventralization of the mouse neural tube. Development 138, 4921-4930, doi:10.1242/dev.070805 (2011).

24 Epstein, D. J., Marti, E., Scott, M. P. \& McMahon, A. P. Antagonizing cAMP-dependent protein kinase $A$ in the dorsal CNS activates a conserved Sonic hedgehog signaling pathway. Development 122, 2885-2894 (1996).

25 Hammerschmidt, M., Bitgood, M. J. \& McMahon, A. P. Protein kinase A is a common negative regulator of Hedgehog signaling in the vertebrate embryo. Genes \& development 10, 647-658, doi:10.1101/gad.10.6.647 (1996).

26 Tiecke, E., Turner, R., Sanz-Ezquerro, J. J., Warner, A. \& Tickle, C. Manipulations of PKA in chick limb development reveal roles in digit patterning including a positive role in Sonic Hedgehog signaling. Developmental biology 305, 312-324, doi:10.1016/j.ydbio.2007.02.017 (2007).

27 Kopinke, D., Roberson, E. C. \& Reiter, J. F. Ciliary Hedgehog Signaling Restricts InjuryInduced Adipogenesis. Cell 170, 340-351 e312, doi:10.1016/j.cell.2017.06.035 (2017).

28 Venugopal, N. et al. The primary cilium dampens proliferative signaling and represses a G2/M transcriptional network in quiescent myoblasts. BMC molecular and cell biology 21, 25, doi:10.1186/s12860-020-00266-1 (2020).

29 Machado, L. et al. In Situ Fixation Redefines Quiescence and Early Activation of Skeletal Muscle Stem Cells. Cell reports 21, 1982-1993, doi:10.1016/j.celrep.2017.10.080 (2017).

30 van Velthoven, C. T. J., de Morree, A., Egner, I. M., Brett, J. O. \& Rando, T. A. Transcriptional Profiling of Quiescent Muscle Stem Cells In Vivo. Cell reports 21, 1994-2004, doi:10.1016/j.celrep.2017.10.037 (2017).

31 Yue, L., Wan, R., Luan, S., Zeng, W. \& Cheung, T. H. Dek Modulates Global Intron Retention during Muscle Stem Cells Quiescence Exit. Developmental cell 53, 661-676 e666, doi:10.1016/j.devcel.2020.05.006 (2020). 
32 Oprescu, S. N., Yue, F., Qiu, J., Brito, L. F. \& Kuang, S. Temporal Dynamics and Heterogeneity of Cell Populations during Skeletal Muscle Regeneration. iScience 23, 100993, doi:10.1016/j.isci.2020.100993 (2020).

33 Katoh, Y. \& Katoh, M. Hedgehog target genes: mechanisms of carcinogenesis induced by aberrant hedgehog signaling activation. Current molecular medicine 9, 873-886, doi:10.2174/156652409789105570 (2009).

34 Yoshida, N., Yoshida, S., Koishi, K., Masuda, K. \& Nabeshima, Y. Cell heterogeneity upon myogenic differentiation: down-regulation of MyoD and Myf-5 generates 'reserve cells'. Journal of cell science 111 ( Pt 6), 769-779 (1998).

35 Tukachinsky, H., Lopez, L. V. \& Salic, A. A mechanism for vertebrate Hedgehog signaling: recruitment to cilia and dissociation of SuFu-Gli protein complexes. The Journal of cell biology 191, 415-428, doi:10.1083/jcb.201004108 (2010).

36 Fan, C. W. et al. The Hedgehog pathway effector smoothened exhibits signaling competency in the absence of ciliary accumulation. Chemistry \& biology 21, 1680-1689, doi:10.1016/j.chembiol.2014.10.013 (2014).

37 Pazour, G. J. et al. Chlamydomonas IFT88 and its mouse homologue, polycystic kidney disease gene $\operatorname{tg} 737$, are required for assembly of cilia and flagella. The Journal of cell biology 151, 709-718, doi:10.1083/jcb.151.3.709 (2000).

38 Rion, N. et al. mTOR controls embryonic and adult myogenesis via mTORC1. Development 146, doi:10.1242/dev.172460 (2019).

39 Le Grand, F., Jones, A. E., Seale, V., Scime, A. \& Rudnicki, M. A. Wnt7a activates the planar cell polarity pathway to drive the symmetric expansion of satellite stem cells. Cell stem cell 4, 535-547, doi:10.1016/j.stem.2009.03.013 (2009).

40 Price, F. D. et al. Inhibition of JAK-STAT signaling stimulates adult satellite cell function. Nature medicine 20, 1174-1181, doi:10.1038/nm.3655 (2014).

41 Dumont, N. A. et al. Dystrophin expression in muscle stem cells regulates their polarity and asymmetric division. Nature medicine 21, 1455-1463, doi:10.1038/nm.3990 (2015).

42 Fu, W., Asp, P., Canter, B. \& Dynlacht, B. D. Primary cilia control hedgehog signaling during muscle differentiation and are deregulated in rhabdomyosarcoma. Proceedings of the National Academy of Sciences of the United States of America 111, 9151-9156, doi:10.1073/pnas.1323265111 (2014).

43 de Morree, A. et al. Staufen1 inhibits MyoD translation to actively maintain muscle stem cell quiescence. Proceedings of the National Academy of Sciences of the United States of America 114, E8996-E9005, doi:10.1073/pnas.1708725114 (2017).

44 Zismanov, V. et al. Phosphorylation of elF2alpha Is a Translational Control Mechanism Regulating Muscle Stem Cell Quiescence and Self-Renewal. Cell stem cell 18, 79-90, doi:10.1016/j.stem.2015.09.020 (2016).

45 Yue, F. et al. Pten is necessary for the quiescence and maintenance of adult muscle stem cells. Nature communications 8, 14328, doi:10.1038/ncomms14328 (2017).

46 Yamaguchi, M. et al. Calcitonin Receptor Signaling Inhibits Muscle Stem Cells from Escaping the Quiescent State and the Niche. Cell reports 13, 302-314, doi:10.1016/j.celrep.2015.08.083 (2015). 
47 Verma, M. et al. Muscle Satellite Cell Cross-Talk with a Vascular Niche Maintains Quiescence via VEGF and Notch Signaling. Cell stem cell 23, 530-543 e539, doi:10.1016/j.stem.2018.09.007 (2018).

48 Zhang, L. et al. The CalcR-PKA-Yap1 Axis Is Critical for Maintaining Quiescence in Muscle Stem Cells. Cell reports 29, 2154-2163 e2155, doi:10.1016/j.celrep.2019.10.057 (2019).

49 Pawlikowski, B., Pulliam, C., Betta, N. D., Kardon, G. \& Olwin, B. B. Pervasive satellite cell contribution to uninjured adult muscle fibers. Skeletal muscle 5, 42, doi:10.1186/s13395-0150067-1 (2015).

50 Keefe, A. C. et al. Muscle stem cells contribute to myofibres in sedentary adult mice. Nature communications 6, 7087, doi:10.1038/ncomms8087 (2015).

51 Zeng, Q. et al. Protective Effects of Sonic Hedgehog Against Ischemia/Reperfusion Injury in Mouse Skeletal Muscle via AKT/mTOR/p70S6K Signaling. Cellular physiology and biochemistry : international journal of experimental cellular physiology, biochemistry, and pharmacology $\mathbf{4 3}$, 1813-1828, doi:10.1159/000484068 (2017).

52 Agarwal, N. K., Qu, C., Kunkalla, K., Liu, Y. \& Vega, F. Transcriptional regulation of serine/threonine protein kinase (AKT) genes by glioma-associated oncogene homolog 1 . The Journal of biological chemistry 288, 15390-15401, doi:10.1074/jbc.M112.425249 (2013).

53 Klein, S. D. et al. Mutations in the sonic hedgehog pathway cause macrocephalyassociated conditions due to crosstalk to the PI3K/AKT/mTOR pathway. American journal of medical genetics. Part A 179, 2517-2531, doi:10.1002/ajmg.a.61368 (2019).

54 Larsen, L. J. \& Moller, L. B. Crosstalk of Hedgehog and mTORC1 Pathways. Cells 9, doi:10.3390/cells9102316 (2020).

55 Feige, P., Brun, C. E., Ritso, M. \& Rudnicki, M. A. Orienting Muscle Stem Cells for Regeneration in Homeostasis, Aging, and Disease. Cell stem cell 23, 653-664, doi:10.1016/j.stem.2018.10.006 (2018).

56 Tierney, M. T. et al. STAT3 signaling controls satellite cell expansion and skeletal muscle repair. Nature medicine 20, 1182-1186, doi:10.1038/nm.3656 (2014).

57 Bentzinger, C. F. et al. Wnt7a stimulates myogenic stem cell motility and engraftment resulting in improved muscle strength. The Journal of cell biology 205, 97-111, doi:10.1083/jcb.201310035 (2014).

58 Renault, M. A. et al. Gli3 regulation of myogenesis is necessary for ischemia-induced angiogenesis. Circulation research 113, 1148-1158, doi:10.1161/CIRCRESAHA.113.301546 (2013).

59 Dumont, N. A., Wang, Y. X. \& Rudnicki, M. A. Intrinsic and extrinsic mechanisms regulating satellite cell function. Development 142, 1572-1581, doi:10.1242/dev.114223 (2015).

60 Piccioni, A. et al. Sonic hedgehog gene therapy increases the ability of the dystrophic skeletal muscle to regenerate after injury. Gene therapy 21, 413-421, doi:10.1038/gt.2014.13 (2014).

61 Piccioni, A. et al. Sonic hedgehog therapy in a mouse model of age-associated impairment of skeletal muscle regeneration. The journals of gerontology. Series A, Biological sciences and medical sciences 69, 245-252, doi:10.1093/gerona/glt076 (2014).

62 Renault, M. A. et al. Hedgehog-dependent regulation of angiogenesis and myogenesis is impaired in aged mice. Arteriosclerosis, thrombosis, and vascular biology 33, 2858-2866, doi:10.1161/ATVBAHA.113.302494 (2013). 
63 Straface, G. et al. Sonic hedgehog regulates angiogenesis and myogenesis during postnatal skeletal muscle regeneration. Journal of cellular and molecular medicine 13, 2424-2435, doi:10.1111/j.1582-4934.2008.00440.x (2009).

64 Hettmer, S. et al. Hedgehog-driven myogenic tumors recapitulate skeletal muscle cellular heterogeneity. Experimental cell research, doi:10.1016/j.yexcr.2015.10.008 (2015).

65 Elia, D., Madhala, D., Ardon, E., Reshef, R. \& Halevy, O. Sonic hedgehog promotes proliferation and differentiation of adult muscle cells: Involvement of MAPK/ERK and PI3K/Akt pathways. Biochimica et biophysica acta 1773, 1438-1446, doi:10.1016/j.bbamcr.2007.06.006 (2007).

66 Xie, L. et al. Transient HIF2A inhibition promotes satellite cell proliferation and muscle regeneration. The Journal of clinical investigation 128, 2339-2355, doi:10.1172/JCI96208 (2018).

67 Judson, R. N. et al. Inhibition of Methyltransferase Setd7 Allows the In Vitro Expansion of Myogenic Stem Cells with Improved Therapeutic Potential. Cell stem cell 22, 177-190 e177, doi:10.1016/j.stem.2017.12.010 (2018).

68 Briggs, D. \& Morgan, J. E. Recent progress in satellite cell/myoblast engraftment -relevance for therapy. The FEBS journal 280, 4281-4293, doi:10.1111/febs.12273 (2013).

69 Blaess, S., Stephen, D. \& Joyner, A. L. Gli3 coordinates three-dimensional patterning and growth of the tectum and cerebellum by integrating Shh and Fgf8 signaling. Development 135, 2093-2103, doi:10.1242/dev.015990 (2008).

70 Murphy, M. M., Lawson, J. A., Mathew, S. J., Hutcheson, D. A. \& Kardon, G. Satellite cells, connective tissue fibroblasts and their interactions are crucial for muscle regeneration. Development 138, 3625-3637, doi:10.1242/dev.064162 (2011).

71 Tallquist, M. D., Weismann, K. E., Hellstrom, M. \& Soriano, P. Early myotome specification regulates PDGFA expression and axial skeleton development. Development 127, 5059-5070 (2000).

72 Srinivas, S. et al. Cre reporter strains produced by targeted insertion of EYFP and ECFP into the ROSA26 locus. BMC developmental biology 1, 4 (2001).

73 Sambasivan, R. et al. Distinct regulatory cascades govern extraocular and pharyngeal arch muscle progenitor cell fates. Developmental cell 16, 810-821, doi:10.1016/j.devcel.2009.05.008 (2009).

74 Smith, L. R. \& Barton, E. R. SMASH - semi-automatic muscle analysis using segmentation of histology: a MATLAB application. Skeletal muscle 4, 21, doi:10.1186/2044-5040-4-21 (2014).

75 Mayeuf-Louchart, A. et al. MuscleJ: a high-content analysis method to study skeletal muscle with a new Fiji tool. Skeletal muscle 8, 25, doi:10.1186/s13395-018-0171-0 (2018).

76 Brun, C. E., Wang, Y. X. \& Rudnicki, M. A. Single EDL Myofiber Isolation for Analyses of Quiescent and Activated Muscle Stem Cells. Methods in molecular biology 1686, 149-159, doi:10.1007/978-1-4939-7371-2_11 (2018).

77 Sincennes, M. C., Wang, Y. X. \& Rudnicki, M. A. Primary Mouse Myoblast Purification using Magnetic Cell Separation. Methods in molecular biology 1556, 41-50, doi:10.1007/978-14939-6771-1_3 (2017).

78 Patro, R., Duggal, G., Love, M. I., Irizarry, R. A. \& Kingsford, C. Salmon provides fast and bias-aware quantification of transcript expression. Nature methods 14, 417-419, doi:10.1038/nmeth.4197 (2017). 
79 Love, M. I., Huber, W. \& Anders, S. Moderated estimation of fold change and dispersion for RNA-seq data with DESeq2. Genome biology 15, 550, doi:10.1186/s13059-014-0550-8 (2014).

80 Zhu, A., Ibrahim, J. G. \& Love, M. I. Heavy-tailed prior distributions for sequence count data: removing the noise and preserving large differences. Bioinformatics 35, 2084-2092, doi:10.1093/bioinformatics/bty895 (2019). 


\section{FIGURES}
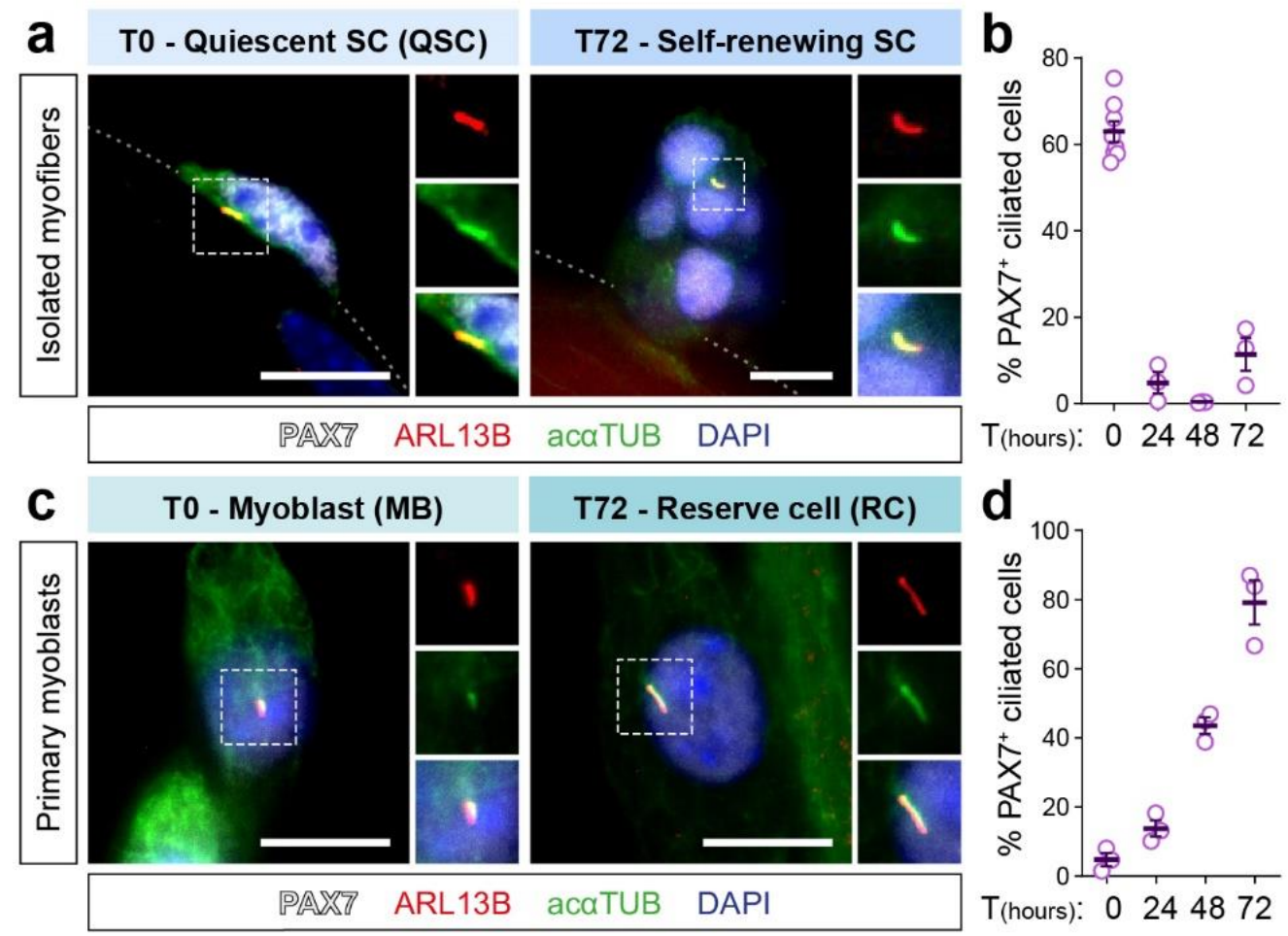

Figure 1. Primary cilia are dynamically regulated in muscle cells.

a) Representative immunofluorescence staining of PAX7 (white), ARL13B (red), acetylated alpha-TUBULIN (acaTUB, green) and nuclei (blue) showing primary cilia on a quiescent satellite cell (SC) from freshly isolated myofiber (T0) and a self-renewing SC from $72 \mathrm{~h}$-cultured myofiber (T72). b) Proportion of PAX7 ${ }^{+}$ satellite cells harboring a primary cilium on freshly isolated myofibers (QSCs, $n=6$ ) and $24 \mathrm{~h}, 48 \mathrm{~h}$ and $72 \mathrm{~h}$ cultured myofibers $(n=3)$. c) Representative immunostaining of PAX7 (white), ARL13B (red), acaTUB (green) and nuclei (blue) showing primary cilia on a myoblast (MB) (T0) and a reserve cell (RC) (T72). d) Proportion of $\mathrm{PAX} 7^{+}$primary myoblasts harboring a primary cilium during $72 \mathrm{~h}$-differentiation time course $(n=3)$.

Scale bars, $10 \mu \mathrm{m}$; Error bars, SEM. 
a

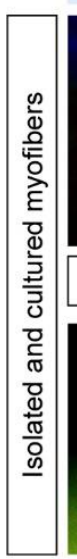

T0

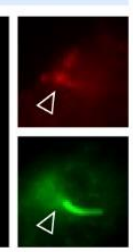

T24

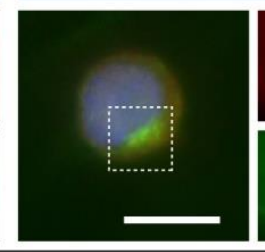

T48
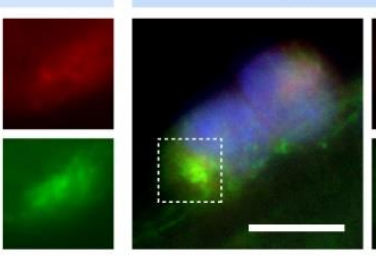
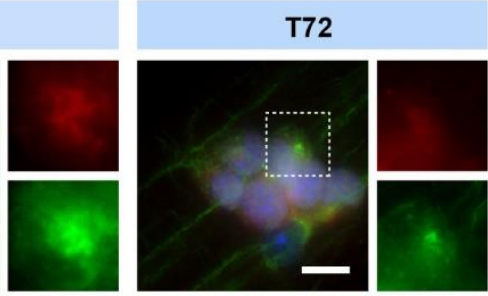

PAAX7 GLI3 acaTUB DAPI
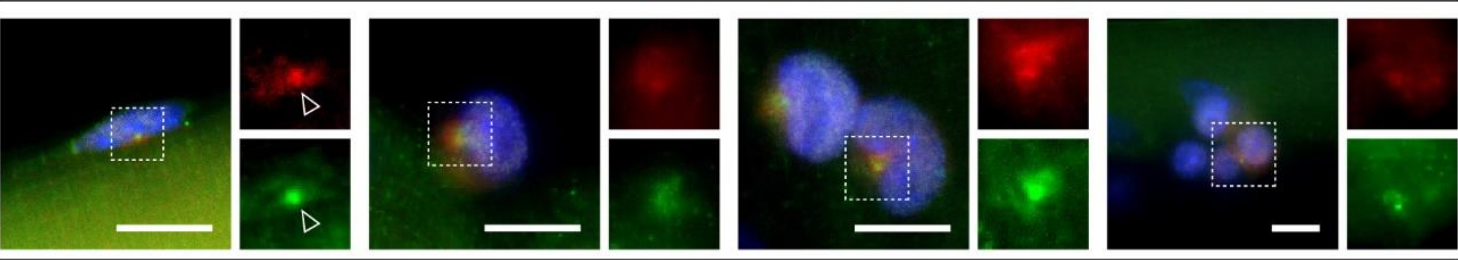

PAXYZ GLI3 PKA DAPI

b

T0

T24

T48

T72
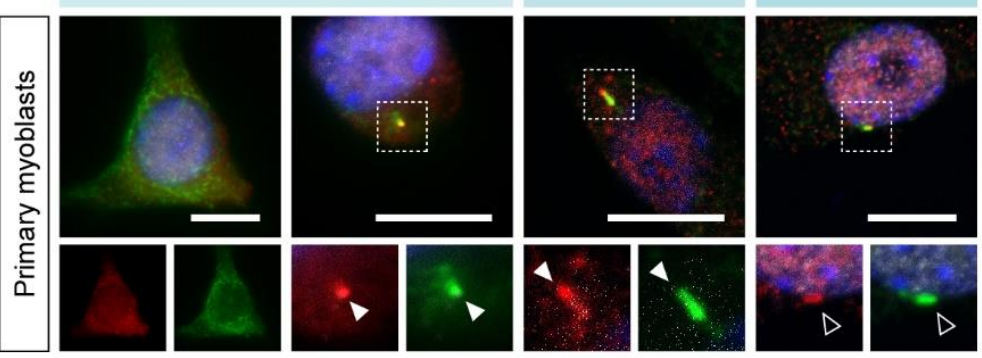

PAXX7 GLI3 acaTUB DAPI
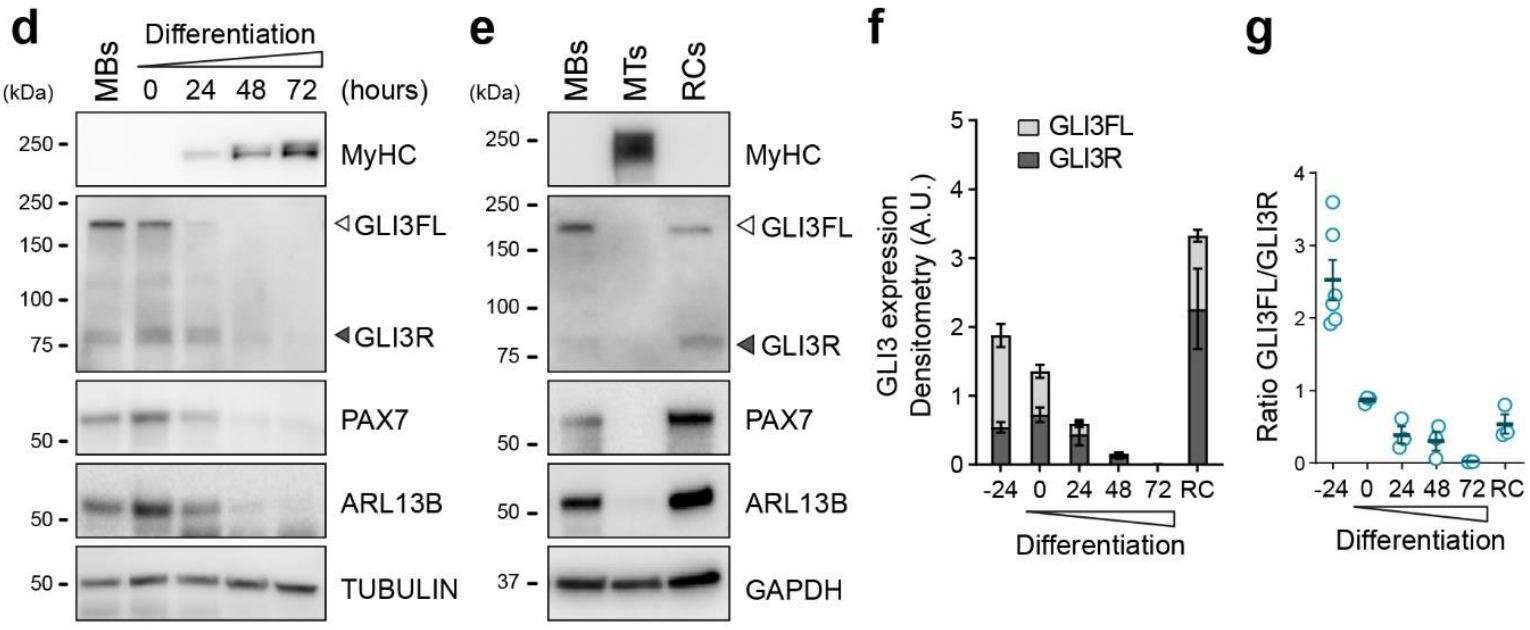

Figure 2. GLI3 subcellular localization and proteolytic processing rely on primary cilia dynamics.

a) Immunostaining showing GLI3 (red) localization in PAX7 ${ }^{+}$(white) quiescent (T0), activated (T24) and proliferating (T48-72) satellite cells on isolated and cultured myofibers. Upper panel, Acetylated $\alpha$-TUBULIN (acaTUB, green) stains the primary cilium (T0) and the microtubule-organizing centers (T24-72). Lower panel, PKA (green) localizes at and around the centrosome areas. b) Immunostaining of GLI3 (red) in primary cilia labeled by acetylated $\alpha$-TUBULIN (acaTUB, green) of $P A X 7^{+}$primary myoblasts (white) during their differentiation time course. c) Proportion of $\mathrm{PAX} 7^{+}$cells that display a GLI3+ primary cilium during myoblast differentiation time course $(n=3)$. d) Immunoblot analysis of the ciliary membrane marker 
ARL13B, GLI3 full-length (GLI3FL) and repressor (GLI3R), in proliferating myoblasts and from 0h to $72 \mathrm{~h}$ of differentiation. PAX7 and MyHC (myosin heavy chain) are used to monitor myogenic differentiation. TUBULIN is used as a loading control. e) Immunoblot analysis of ARL13B, GLI3FL and GLI3R in myoblasts (MBs), 72h-differentiated myoblasts or myotubes (MTs) and reserve cells (RCs). Both MBs and RCs express PAX7 while MTs express the myogenic differentiation marker MyHC. GAPDH is used as a loading control. f) Densitometric analysis of the level of GLI3FL and GLI3R relative to TUBULIN signals of $6(-24 \mathrm{~h})$ and 3 biological replicates (0h-72h). g) Ratio of GLI3FL/GLI3R relative to GAPDH $(-24 h, n=6 ; 0 h-72 h$, $\mathrm{n}=3$ ).

White arrows indicate the tip of the primary cilium; Empty arrows indicate the basal body; Scale bar, 10 $\mu m$; Error bars, SEM. 

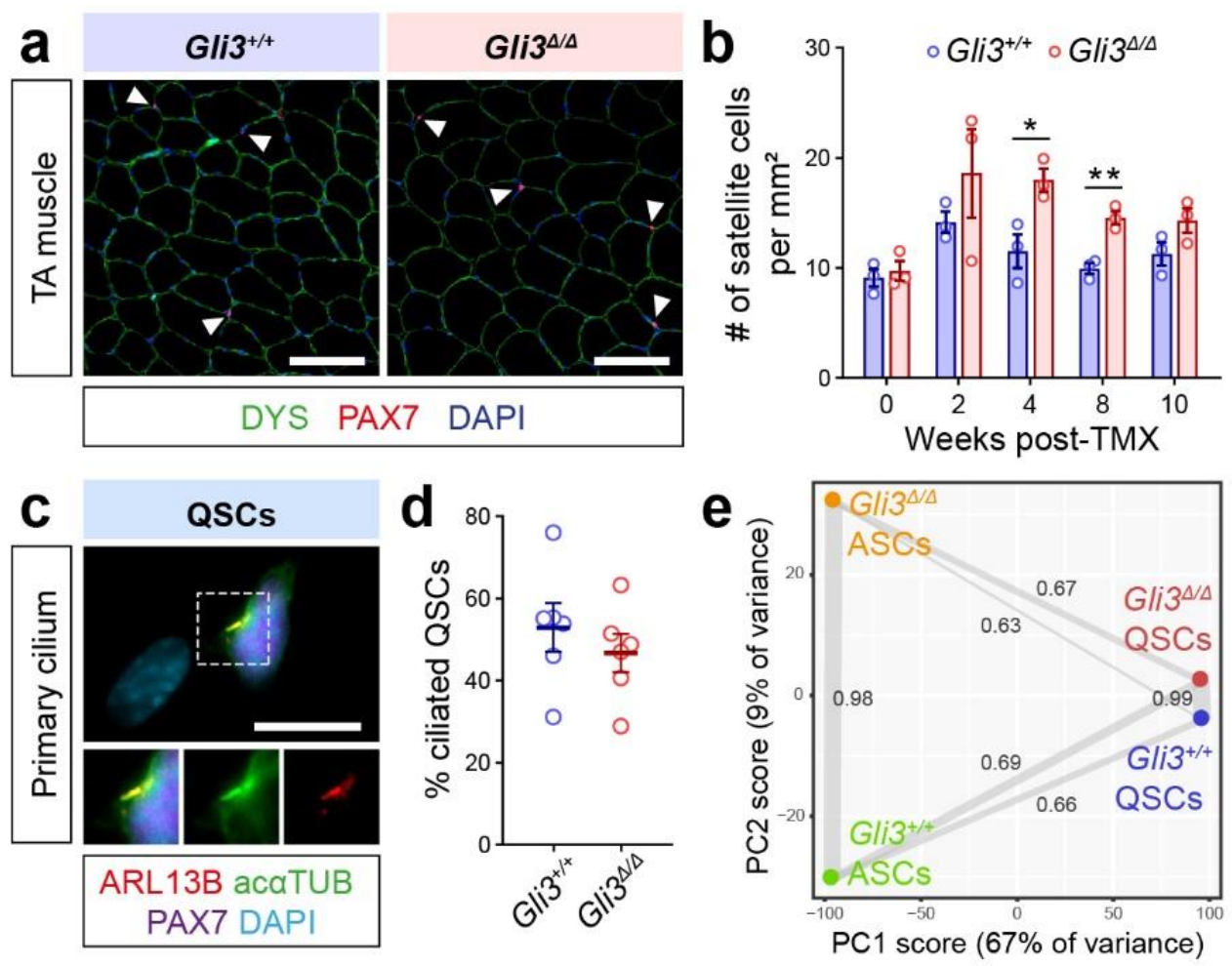

\section{Figure 3. GLI3 controls the quiescence of satellite cells.}

a) Representative immunofluorescence picture showing transversal cross-sections of resting TA muscle in $\mathrm{Gli}^{+/+}$and Gli3 ${ }^{\mathrm{L} \Delta}$ mice. PAX7 (red) labels the satellite cells and DYS (green) delineates the myofibers. Nuclei are stained with DAPI (blue). Scale bars represent $50 \mu \mathrm{m}$. b) Quantification of the number of PAX7 ${ }^{+}$ satellite cells per $\mathrm{mm}^{2}$ in resting TA muscle from $\mathrm{Gli}^{+/+}$and $\mathrm{Gli} 3^{\mathrm{L} / \Delta}$ mice following tamoxifen treatment ( $n=3$ males). c) Primary cilia are immunostained with ARL13B (red) and ac $\alpha$ TUB (green) and satellite cells with PAX7 (purple). Nuclei are labeled with DAPI (blue). Scale bars represent 10 $\mu \mathrm{m}$. d) Proportions of ciliated satellite cells on freshly isolated EDL myofibers from Gli3 $3^{+++}$and Gli3 $3^{\Delta \Delta}$ mice $(n=6,3$ males and 3 females for each genotype). e) Principal component analysis (PCA) of global transcriptomes of $\mathrm{Gli3^{+/+ }}$ and Gli3 $^{\mathrm{L} / \Delta}$ quiescent (QSCs) and activated satellite cells (ASCs) and Pearson's values showing the correlation between samples. Each dot represents the mean of 3 biological samples.

Error bars, SEM; ${ }^{*} p<0.05 ;{ }^{* * *} p<0.001$ 
bioRxiv preprint doi: https://doi.org/10.1101/2020.12.07.415273; this version posted December 7, 2020. The copyright holder for this preprint (which was not certified by peer review) is the author/funder. All rights reserved. No reuse allowed without permission.

a GO terms - Up-regulated genes in $\mathrm{Gli}^{\Delta / \Delta} \mathrm{vs} \mathrm{Gli3^{+/+ }} \mathrm{QSCs}$

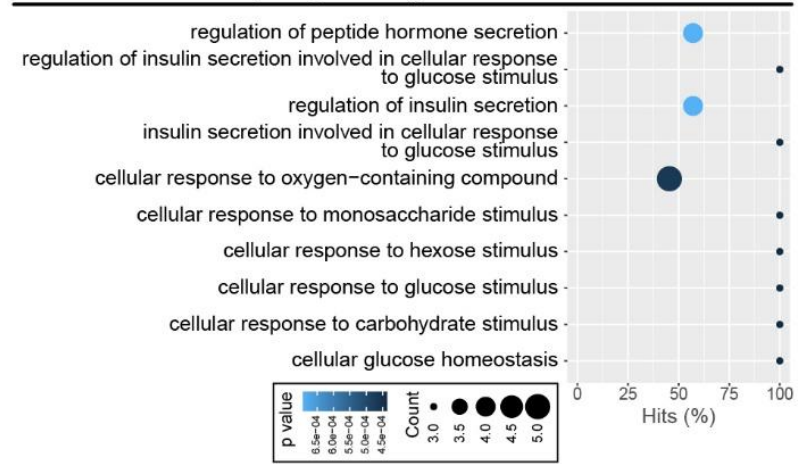

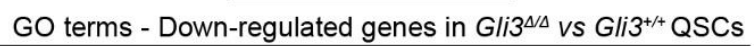

regulation of skeletal muscle cell differentiation

positive regulation of T-helper cell differentiation

defense response to other organism

defense response to bacterium

cellular response to oxygen-containing compound

cellular response to oxidative stress

arachidonic acid secretion

arachidonate transport

antimicrobial humoral response

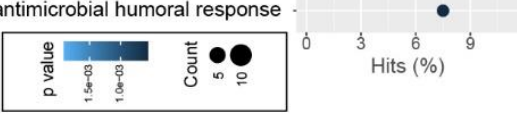

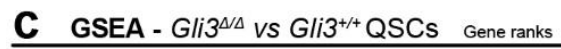

HALLMARK_OXIDATIVE_PHOSPHORYLATION HALLMARK_INTERFERON_ALPHA_RESPONSE -

HALLMARK_PROTEIN_SECRETION '-

HALLMARK_FATTY_ACID_METABOLISM HALLMARK_DNA_REPAIR -

HALLMARK_APICAL_SURFACE -

HALLMARK_PEROXISOME -

HALLMARK_INTERFERON_GAMMA_RESPONSE -

HALLMARK_MTORC1_SIGNALING -

HALLMARK_ADIPOGENESIS -

HALLMARK_KRAS_SIGNALING_UP -

HALLMARK_UV_RESPONSE_UP -

HALLMARK_G2M_CHECKPOINT "

HALLMARK_IL2_STAT5_SIGNALING -

HALLMARK_TNFA_SIGNALING_VIA_NFKB

HALLMARK_WNT_BETA_CATENIN_SIGNALING

HALLMARK_ANGIOGENESIS

HALLMARK_NOTCH_SIGNALING

HALLMARK_IL6_JAK_STAT3_SIGNALING

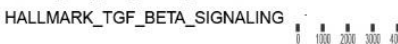

b GO terms - Up-regulated genes in $\mathrm{Gli}^{\Delta / \Delta}$ vs Gli $3^{+/+} \mathrm{ASCs}$

regulation of $\mathrm{G} 1 / \mathrm{S}$ transition of mitotic cell cycle

regulation of cell cycle $\mathrm{G} 1 / \mathrm{S}$ phase transition positive regulation of cellular process

positive regulation of cell projection organization positive regulation of biological process

negative regulation of $\mathrm{G} 1 / \mathrm{S}$ transition of mitotic cell cycle

negative regulation of cell cycle G1/S phase transition $\mathrm{G} 1 / \mathrm{S}$ transition of mitotic cell cycle DNA repair

cellular response to DNA damage stimulus . cell cycle $\mathrm{G} 1 / \mathrm{S}$ phase transition

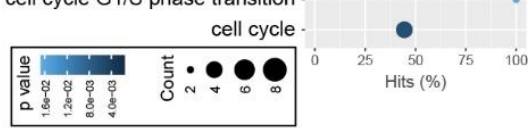

GO terms - Down-regulated genes in Gli3 ${ }^{\Delta / \Delta}$ vs $\mathrm{Gli3}^{+/+} \mathrm{ASCs}$

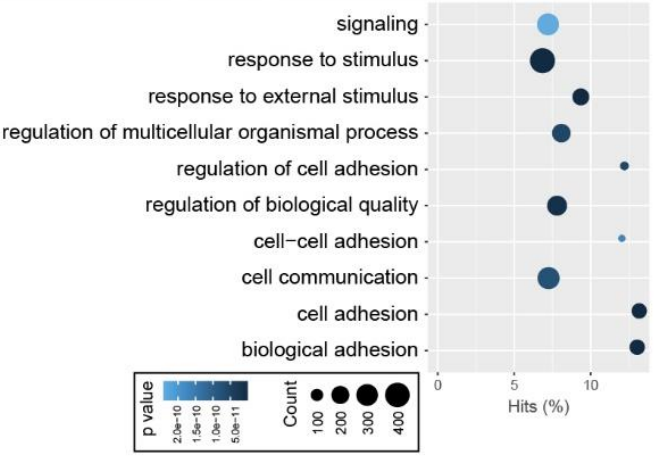

d GSEA - Gli3 ${ }^{\Delta / \Delta}$ vs Gli3 ${ }^{+/+}$ASCS Gene ranks NES pval padj $\begin{array}{llll}\text { HALLMARK_MYC_TARGETS_V1 } & 3.51 & 6.2 \mathrm{e}-02 & 1.0 \mathrm{e}-01\end{array}$ HALLMARK_E2F_TARGETS $\cdots \cdots \cdots$

HALLMARK_OXIDATIVE_PHOSPHORYLATION —-1..... $2.80 \quad 5.6 \mathrm{e}-02 \quad 9.6 \mathrm{e}-02$ HALLMARK_MYC_TARGETS_V2 HALLMARK_GPM_CHECKPOINT — TAL_MARK_DNA_REPAR

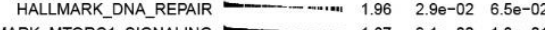
HALLMARK_MTORC1_SIGNALING $\longrightarrow 1.67 \quad 9.1 \mathrm{e}-02 \quad 1.3 \mathrm{e}-01$ HALLMARK_UNFOLDED_PROTEIN_RESPONSE - _.-... $1.49 \quad 1.8 \mathrm{e}-02 \quad 4.3 \mathrm{e}-02$

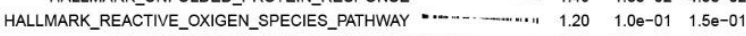
HALLMARK_FATTY_ACID_METABOLISM - $1.18 \quad 6.1 \mathrm{e}-02 \quad 1.0 \mathrm{e}-01$ HALLMARK_IL2_STAT5_SIGNALING $\cdots\left[\begin{array}{ccc}-1.57 & 1.0 \mathrm{e}-03 & 4.0 \mathrm{e}-03\end{array}\right.$ HALLMARK APICAL JUNCTION *..-_ - $-1.00-1.00-03-4.0 e-03$ HALLMARK_TNFA_SIGNALING_VIA_NFKB $-\begin{array}{llll}-1.70 & 1.0 \mathrm{e}-03 & 4.0 \mathrm{e}-03\end{array}$

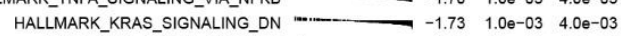

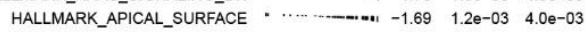
HALLMARK_KRAS_SIGNALING_UP $-\begin{array}{lll}-1.90 & 1.0 \mathrm{e}-03 & 4.0 \mathrm{e}-03\end{array}$ HALLMARK_EPITHELIAL_MESENCHYMAL_TRANSITION $1 \cdots-\cdots-1.91 \quad 1.0 \mathrm{e}-03 \quad 4.0 \mathrm{e}-03$

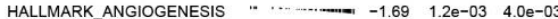
HALLMARK INFLAMMATORY RESPONSE :- $\quad-2.05 \quad 1.0 \mathrm{e}-03 \quad 4.0 \mathrm{e}-03$ HALLMARK_INTERFERON_GAMMA_RESPONSE $\quad-1 . .-2.08 \quad 1.00-03 \quad 4.0 \mathrm{e}-03$

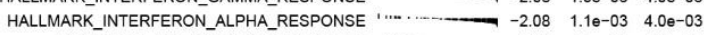

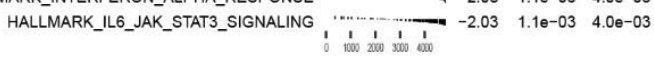

\section{Figure 4. Gli3 deletion in satellite cells activates mTORC1 signaling.}

a) GO term analysis Gene Ontology (GO) term enrichment (Biological process) for the up-regulated and

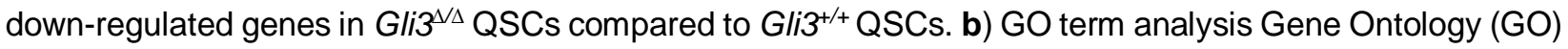
term enrichment (Biological process) for the up-regulated and down-regulated genes in Gli3 ${ }^{\Delta / \Delta}$ ASCs

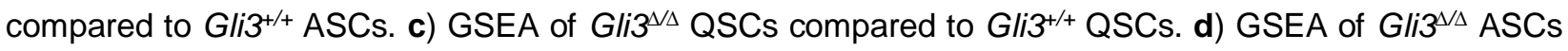
compared to $\mathrm{Gli3}^{+/+}$ASCs. 
a

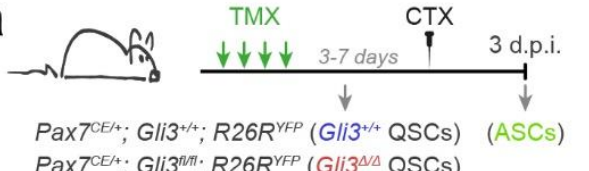

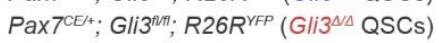

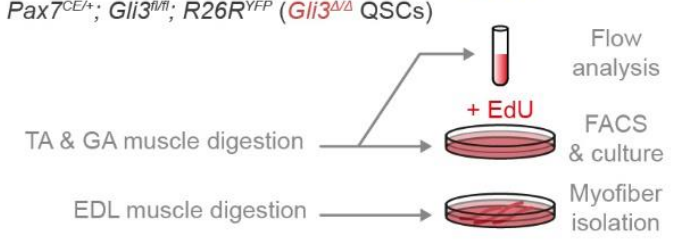

d

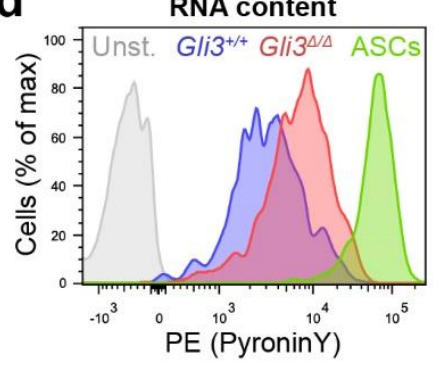

h

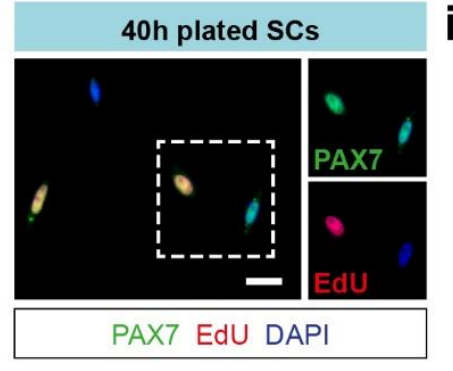

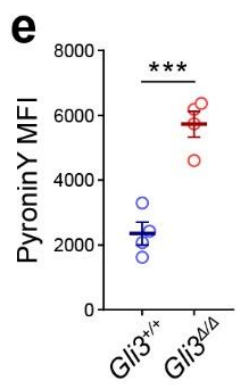

i

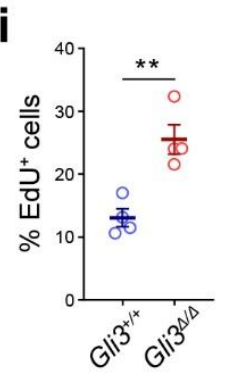

b

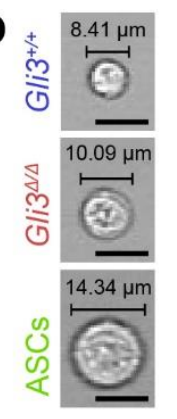

C

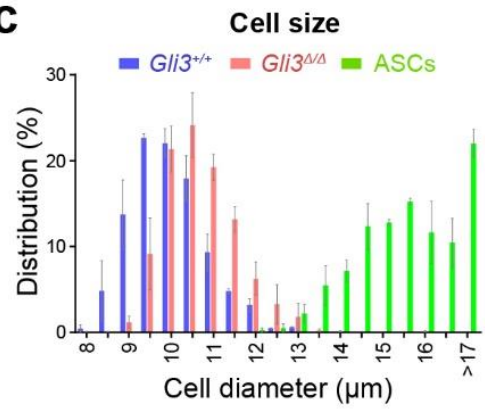

f
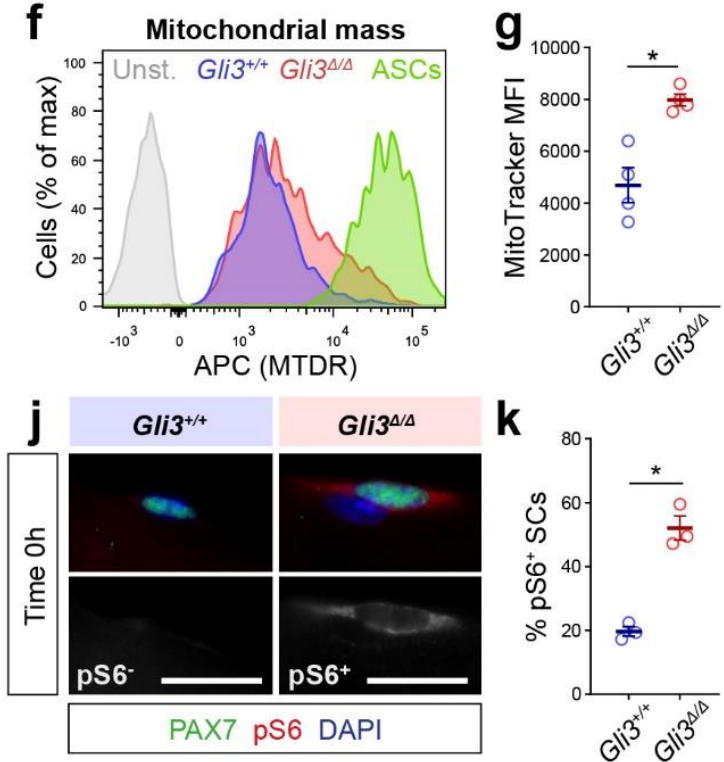

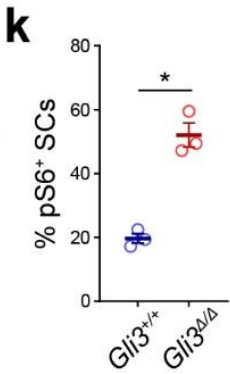

Figure 5. Satellite cells in Gli3 ${ }^{\Delta / \Delta}$ uninjured muscle are in $\mathrm{G}_{\text {Alert }}$

a) Experimental design. Satellite cells (SCs) from TA and gastrocnemius (GA) muscles were analyzed for cell size, RNA content and mitochondrial mass. FACS-isolated SCs were plated 40h with EdU to analyze cell cycle entry. Phosphorylation status of S6 ribosomal protein was analyzed on freshly isolated EDL myofibers. b) Representative images of freshly sorted $\mathrm{Gli3}^{+/+}$and $\mathrm{Gli}^{\mathrm{L} / \Delta}$ quiescent satellite cells (QSCs)

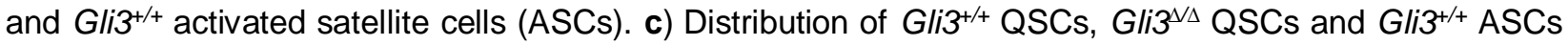
according to their diameter ( $\mathrm{n}=3$ males, $>100$ cells per mouse). d) Representative flow cytometry plot of Gli3 $^{+/+}$QSCs, Gli3 ${ }^{\Delta / \Delta}$ QSCs and Gli3 ${ }^{+/+}$ASCs stained for PyroninY (Unst. = unstained). e) Mean fluorescence intensity (MFI) of PyroninY staining in Gli3 ${ }^{+/+}$and Gli3 ${ }^{\Delta / \Delta}$ QSCs ( $n=4$ males). $f$ ) Representative

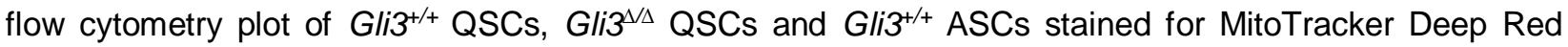
(MTDR). g) MFI of MitoTracker staining in Gli3 ${ }^{+/+}$and Gli3 ${ }^{\Delta / \Delta}$ QSCs ( $n=4$ males). $\mathbf{h}$ ) Immunostaining of SCs with PAX7 (green) and DAPI (blue) showing EdU (red) incorporation 40h post-isolation. i) Proportion of $\mathrm{EdU}^{+} \mathrm{SCs}$ in $\mathrm{Gli}^{+/+}$and Gli3 ${ }^{\Delta / \Delta}$ mice ( $\mathrm{n}=4,3$ males and 1 female). j) Immunofluorescence of phospho-S6

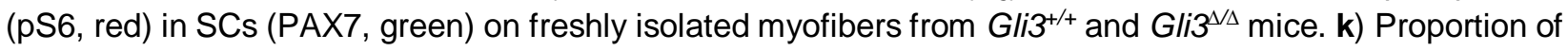
$\mathrm{pS6}^{+}$SCs in $\mathrm{Gli3}^{+/+}$and Gli3 ${ }^{\mathrm{V} \Delta}$ mice ( $\mathrm{n}=3$ females).

Scale bars, $10 \mu \mathrm{m}$; Error bars, SEM; ${ }^{*} p<0.05 ;{ }^{* \star} p<0.01 ;{ }^{* \star} p<0.001$. 
a
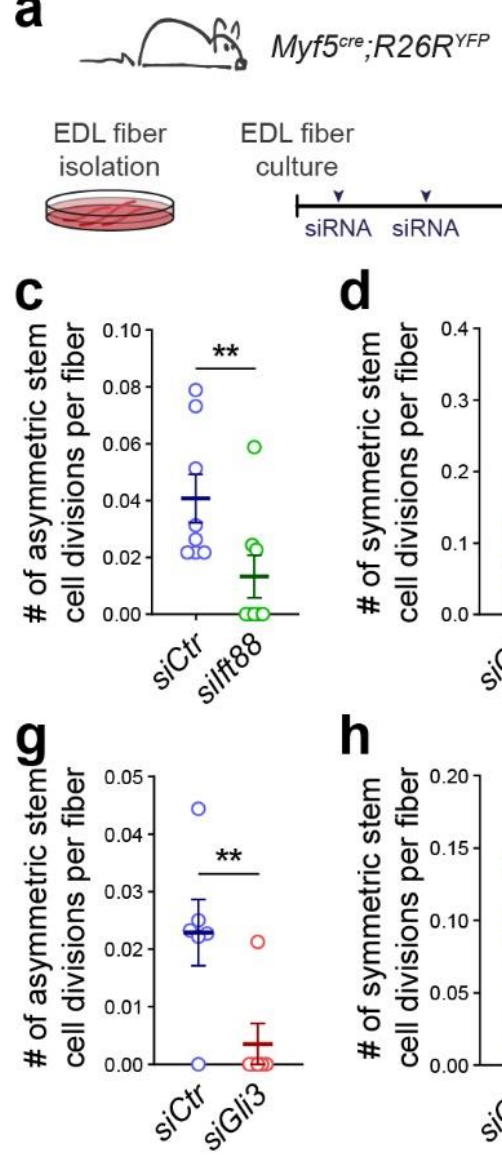

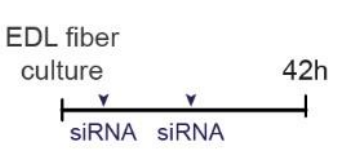

d

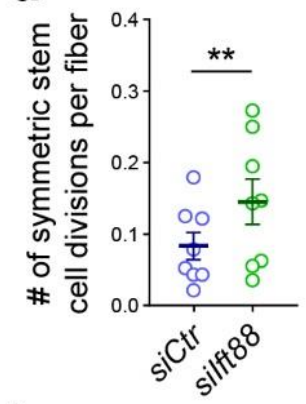

h

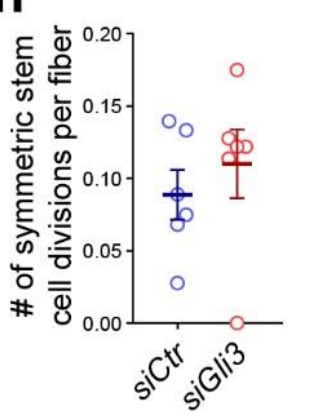

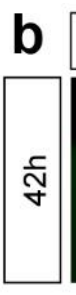

e
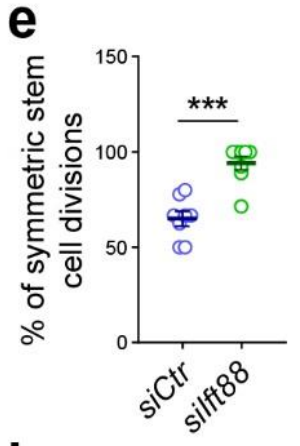

i

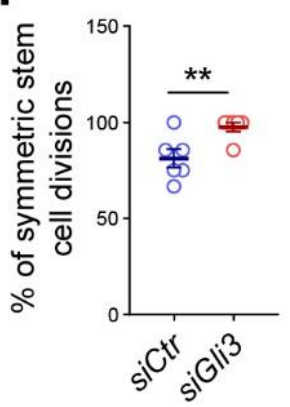

PAX7 YFP DAPI

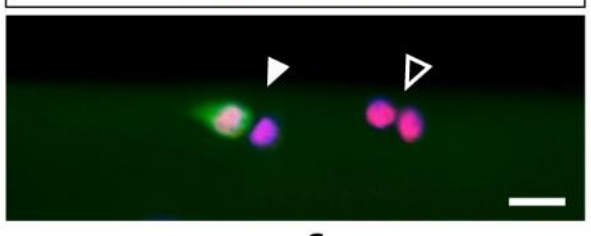

f
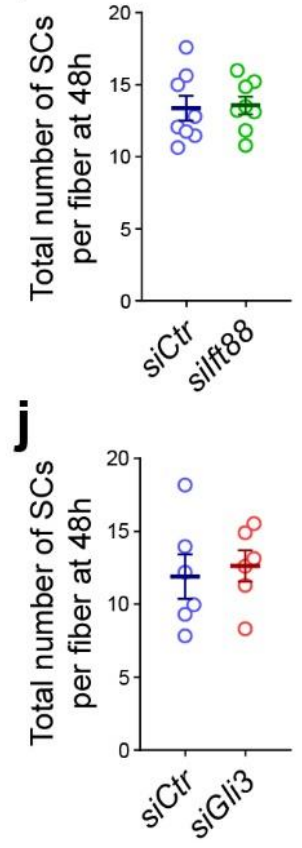

Figure 6. Primary cilia-mediated GL/3 processing regulates asymmetric division.

a) Experimental design followed to knockdown Gli3 or Ift88 in satellite cells on cultured Myf5cre:R26R ${ }^{\text {YFP }}$ single EDL myofibers. b) Representative immunofluorescence staining of PAX7 (red), YFP (green) and nuclei (DAPI, blue) showing an asymmetric (white arrow) and a symmetric (empty arrow) stem cell division on a $M y f 5^{c r e}: R 26 R^{Y F P}$ EDL myofiber cultured for $42 \mathrm{~h}$. c) Quantification of asymmetric stem cell doublets per fiber following treatment with siRNA against Ift88 (silft88) or with a non-target control (siCtr) ( $\mathrm{n}=8$ males). d) Quantification of symmetric stem cell divisions per fiber following treatment with silft88 or $\operatorname{siCtr}(\mathrm{n}=8$ males). e) Proportion of symmetric stem cell divisions following treatment with silft88 or $\operatorname{siCtr}(\mathrm{n}=8$ males). f) Total number of $\mathrm{PAX7} 7^{+}$satellite cells per fiber following treatment with siRNA against Ift88 (silft88) or with a non-target control (siCtr) ( $\mathrm{n}=8$ males). g) Quantification of asymmetric stem cell divisions per fiber following treatment with siRNA against Gli3 (siGli3) or with siCtr ( $\mathrm{n}=6$ males). $\mathrm{h}$ ) Quantification of symmetric stem cell doublets per fiber following treatment with siGli3 or $\operatorname{siCtr}(\mathrm{n}=6$ males). i) Proportion of symmetric stem cell divisions following treatment with siGli3 or $\operatorname{siCtr}(\mathrm{n}=6$ males). $\mathrm{j})$ Total number of PAX7 ${ }^{+}$ satellite cells per fiber following treatment with siRNA against Gli3 (siGli3) or with a non-target control (siCtr) $(\mathrm{n}=6$ males).

Scale bar, $10 \mu \mathrm{m}$; Error bars, SEM; ${ }^{* *} p<0.01 ;{ }^{* * *} p<0.001$. 

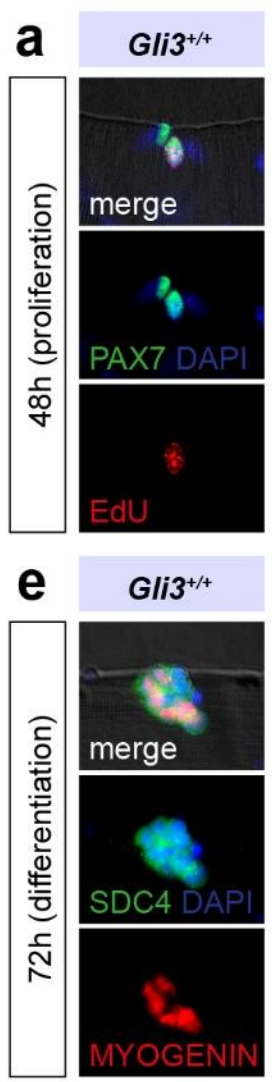

b
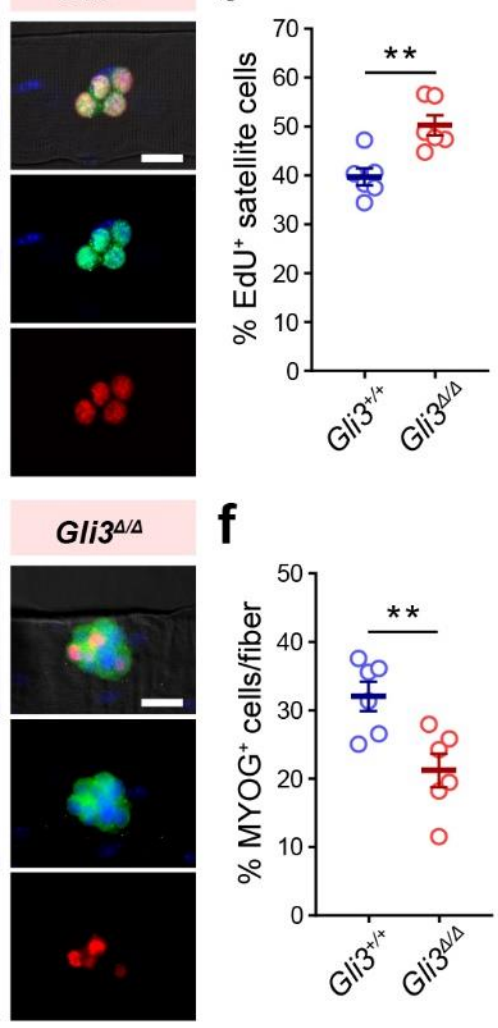

C

\section{ASCs}

Lin- (CD45, CD11b, Sca1, CD31) ITGA7+ VCAM+

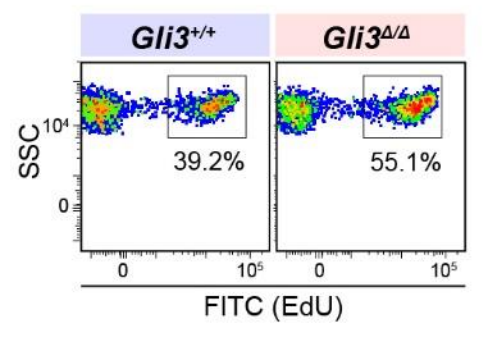

d
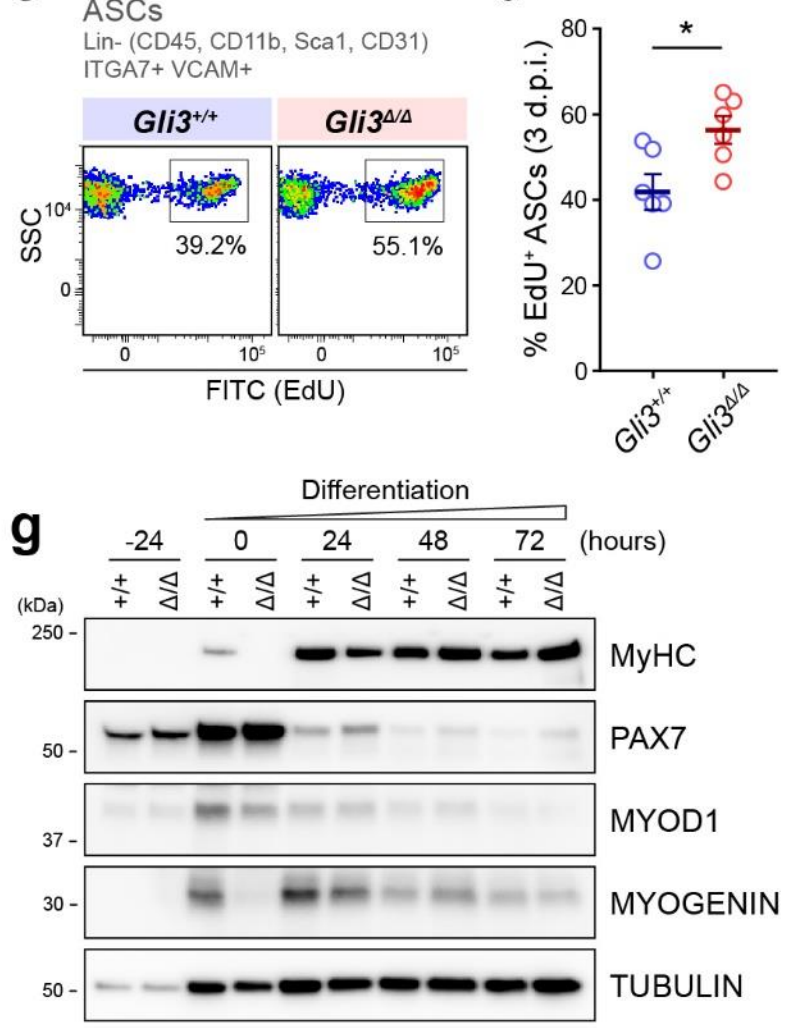

Figure 7. Gli3 deletion allows for the expansion of the satellite cell pool.

a) Satellite cell immunostaining with PAX7 (green) and DAPI (nuclei, blue) showing EdU (red) incorporation by the $\mathrm{PAX}^{+}$cells after $1 \mathrm{~h}$ EdU incubation. b) Proportion of $\mathrm{Gli} 3^{+/+}$and Gli3 ${ }^{\mathrm{\Delta} / \Delta}$ satellite cells that have incorporated EdU (EdU $\left.{ }^{+}\right)$. c) Representative flow cytometry plot of $\mathrm{Gli}^{+/+}$and $\mathrm{Gli} \mathrm{3}^{\mathrm{\Delta} / \Delta}$ activated satellite cells (ASCs) that have incorporated EdU. d) Proportion of $\mathrm{EdU}^{+}$Gli3 ${ }^{+/+}$and $\mathrm{Gli} 3^{\Delta / \Delta}$ ASCs isolated from injured muscles at 3 days post-injury (d.p.i.). e) Representative immunofluorescence using anti-SYNDECAN-4 (SDC4, green) to label all satellite cells and MYOGENIN (MYOG, red) to mark the differentiated ones. DAPI stains the nuclei in blue. f) Quantification of the number of $\mathrm{MYOG}^{+}$satellite cells per $\mathrm{Gli}^{+++}$and $\mathrm{Gli}^{\mathrm{N} / \Delta}$ myofiber after $72 \mathrm{~h}$ of culture. g) Immunoblot analysis of MyHC, PAX7, MYOD1 and MYOGENIN from Gli3 ${ }^{+/+}$ and $\mathrm{Gli}^{\mathrm{L} / \Delta}$ myoblasts differentiated for $72 \mathrm{~h}$. TUBULIN is used as a loading control.

Unless indicated otherwise, $\mathrm{n}=6$ ( 3 males and 3 females for each genotype); Scale bars, $10 \mu \mathrm{m}$; Error bars, SEM; ${ }^{*} p<0.05 ;{ }^{* *} p<0.01$ 


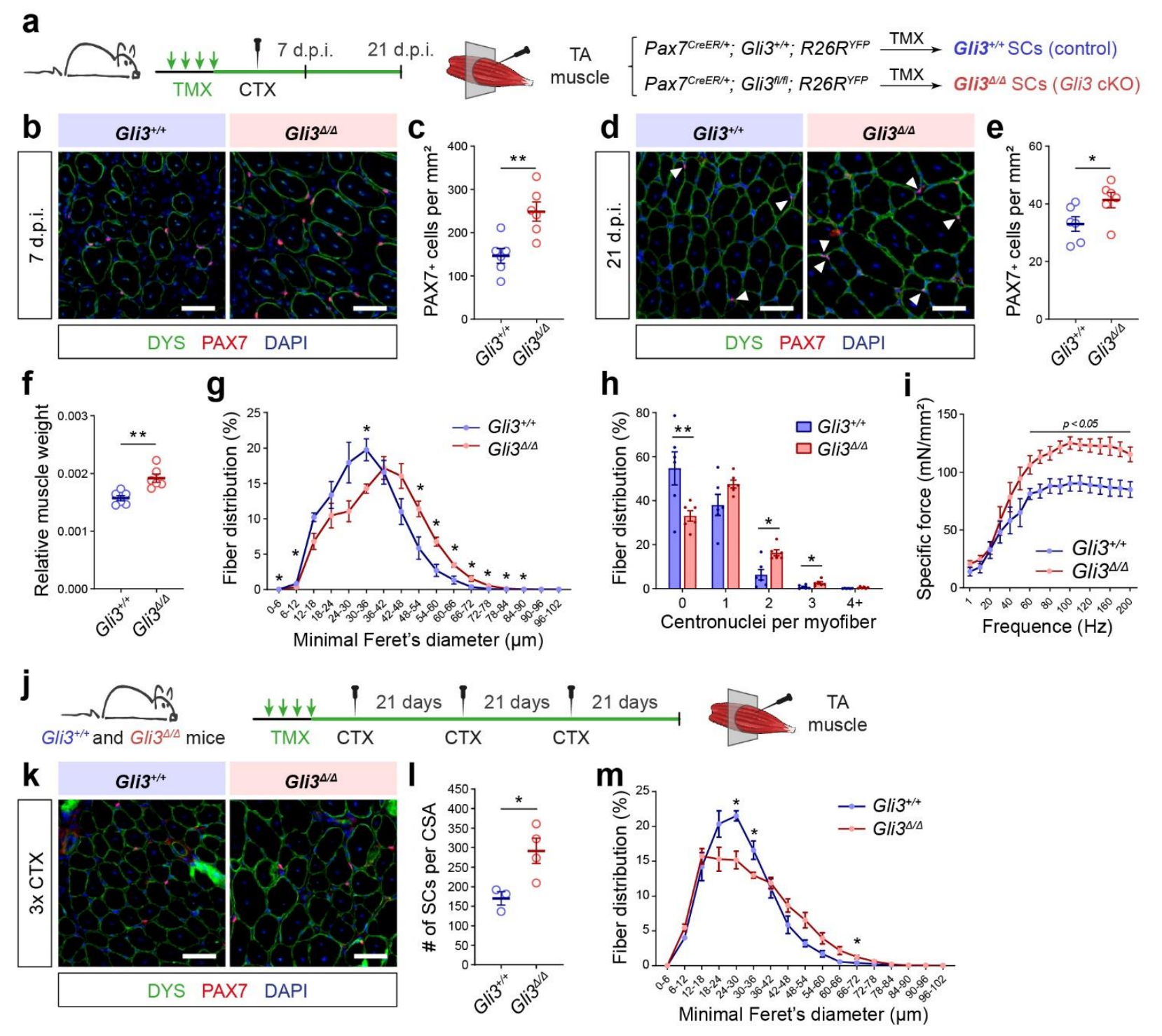

Figure 8. Gli3 deletion in satellite cells enhances muscle regeneration.

a) Experimental design to analyze muscle regeneration of $G / i 3^{+/+}$and Gli3 ${ }^{\mathrm{L} \Delta}$ mice. Muscle injury was induced by cardiotoxin (CTX) injections. b) Representative images of TA muscle sections at 7 days postinjury (d.p.i.). Satellite cells (SCs) are labelled with PAX7 (red). DYSTROPHIN (DYS, green) delineates the regenerating myofibers. c) Quantification of PAX7 $7^{+}$SCs per $\mathrm{mm}^{2} \mathrm{TA}$ section of $\mathrm{Gli} 3^{+/+}$and $\mathrm{Gli} 3^{\mathrm{V} \Delta}$ mice at 7 d.p.i. d) Immunostaining of PAX7 and DYSTROPHIN on TA muscle sections at 21 d.p.i. e) Quantification of PAX7+ SCs per mm² TA section, $\mathbf{f}$ ) TA muscle weight normalized to total body weight, $\mathbf{g}$ ) distribution of TA myofibers according to minimal Feret's diameter and $\mathbf{h}$ ) distribution of regenerated myofibers according to their number of centrally located nuclei (centronuclei) of $\mathrm{Gli} 3^{+/+}$and $\mathrm{Gli} 3^{\mathrm{N} / \Delta}$ mice at 21 d.p.i. $\mathrm{n}=6$ (3 males and 3 females) i) Specific force of regenerated TA muscles from Gli3 ${ }^{+/+}$and Gli3 ${ }^{\Delta / \Delta}$ mice at 21 d.p.i. $\left(n=5\right.$ males). j) Experimental design to analyze regeneration of $G / i 3^{+/+}$and Gli3 ${ }^{\Delta / \Delta}$ mice following three consecutive CTX-injuries in the TA muscle (3XCTX). k) Immunostaining of PAX7 and DYSTROPHIN on TA muscle sections at following 3xCTX. I) Quantification of $P A X 7^{+}$satellite cells per cross-sectional area (CSA) of injured TA and $\mathbf{m}$ ) distribution of regenerated TA myofiber according to minimal Feret's diameter of 3xCTX $\mathrm{Gli}^{+/+}$and $\mathrm{Gli} 3^{\mathrm{L} / \Delta}$ mice.

DAPI stains nuclei (blue); Scale bars, $50 \mu \mathrm{m}$; Error bars, SEM; ${ }^{*} p<0.05 ;{ }^{*} p<0.01$ 


\section{SUPPLEMENTARY FIGURES}
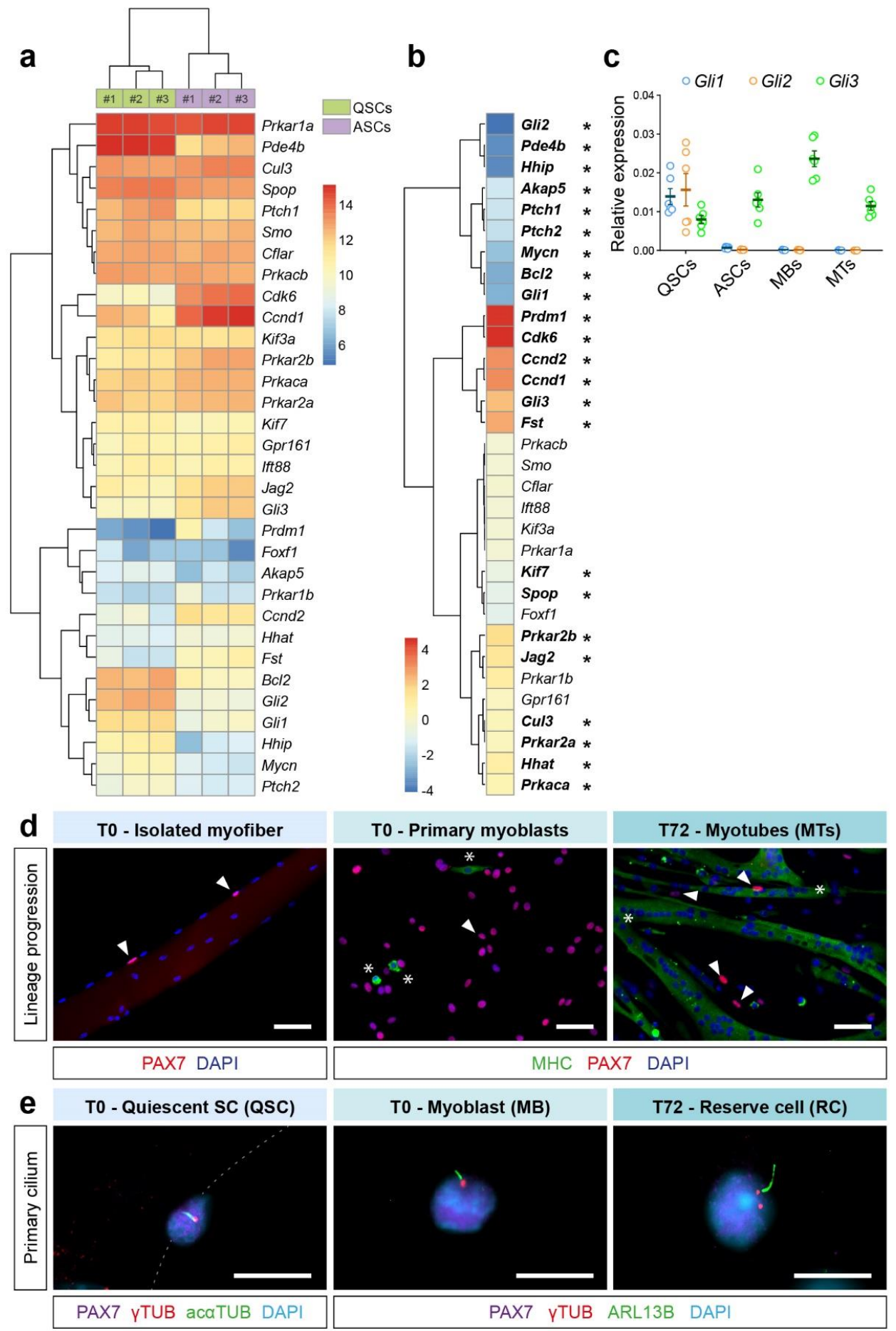

\section{Supplementary Figure 1. Gli3 is expressed in muscle cells throughout their lineage progression.}

a) Heatmap from normalized and log2 transformed expression matrix of components and target genes of canonical Hedgehog signaling in quiescent (QSCs) and activated satellite cells (ASCs) ( $n=3$ males). b) Heatmap showing fold change values of canonical Hedgehog signaling genes in ASCs compared to QSCs. Significantly up- and down-regulated genes are written in bold with a star that indicates $p$ adjusted values 
(padj) < 0.05. c) Expression of Gli1, Gli2 and Gli3 determined by RT-qPCR and normalized to Ppia and Rps18 in QSCs, ASCs, myoblasts (MBs) and 3 days-differentiated muscle cells or myotubes (MTs) $(n=6$ (3 males and 3 females for each genotype)). d) Representative immunofluorescence staining of PAX7 (red) labelling quiescent satellite cells on an isolated myofiber (TO - Isolated myofiber), and primary myoblasts (T0 - Primary myoblasts) and reserve cells appearing along the myotubes (T72 - Myotubes). Myosin heavy chain (MyHC, green, asterisks) labels differentiated muscle cells or myotubes (MTs). e) Representative immunostaining of gamma-TUBULIN (YTUB, red) and acetylated alpha-TUBULIN (acaTUB, green) showing the basal body and primary cilium on a $P A X 7^{+}$quiescent satellite cell (purple, T0 - QSC). YTUB (red) and $A R L 13 B$ (green) label respectively the basal body and primary cilium of both PAX7 $7^{+}$myoblast (purple, $\mathrm{T}-\mathrm{MB}$ ) and reserve cell (purple, T72 - RC). Nuclei (blue) are labelled with DAPI.

Scale bars, $10 \mu \mathrm{m}$; Error bars, SEM; ${ }^{*} p<0.05$. 

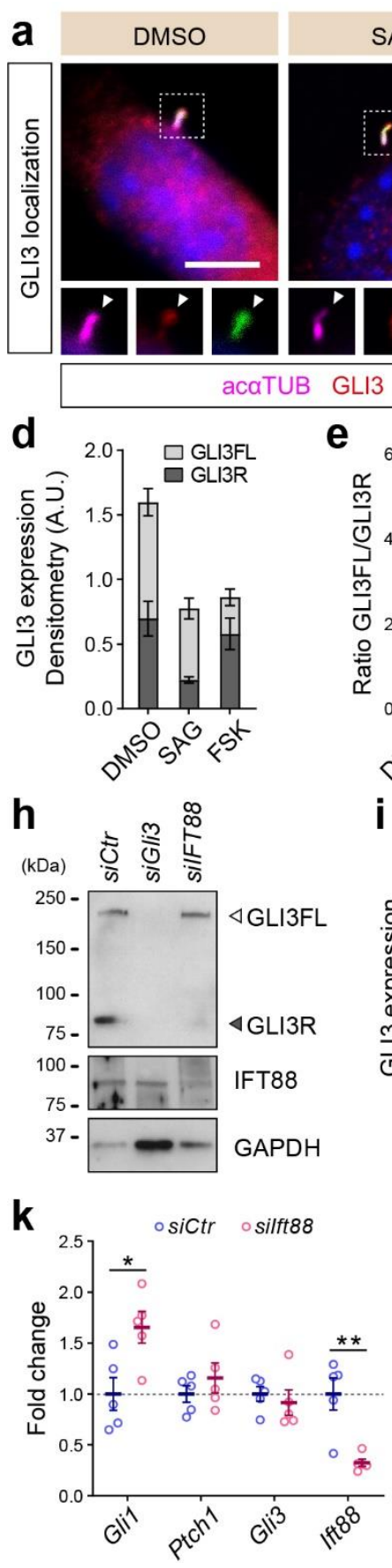

$$
\text { SAG }
$$
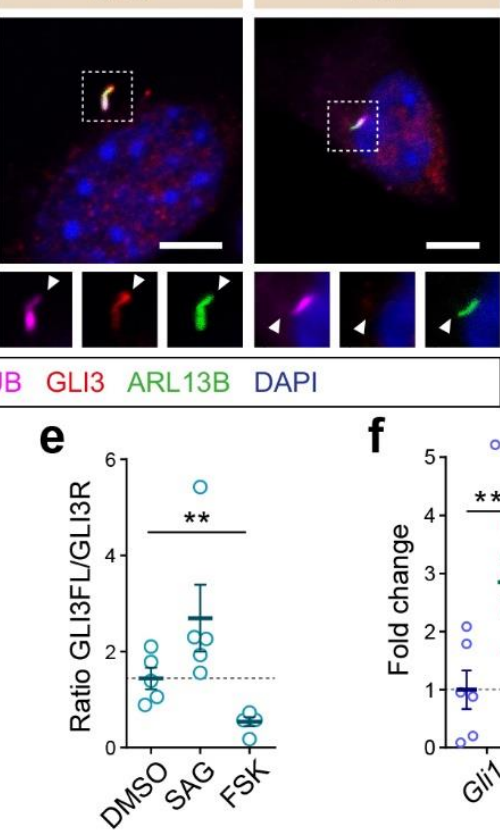

i

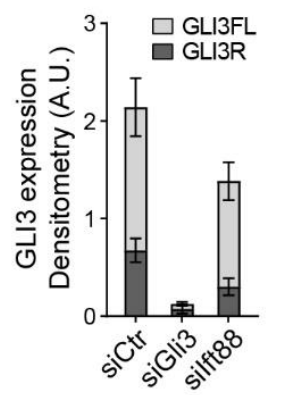

j

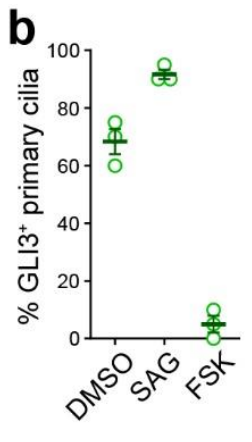

f ${ }^{5}$ ○DMSO $\circ$ SAG
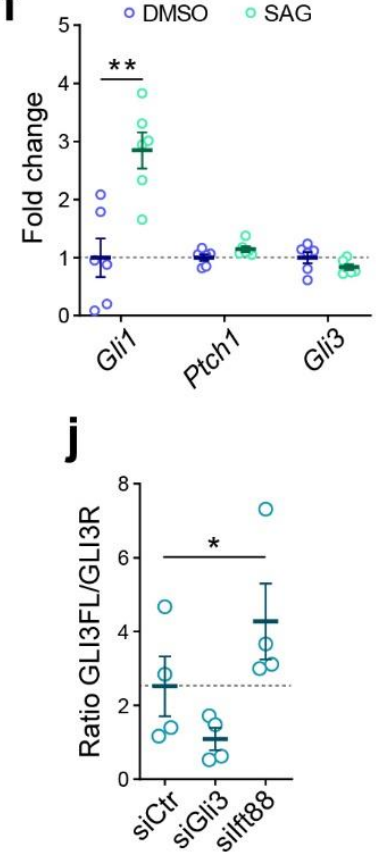

I
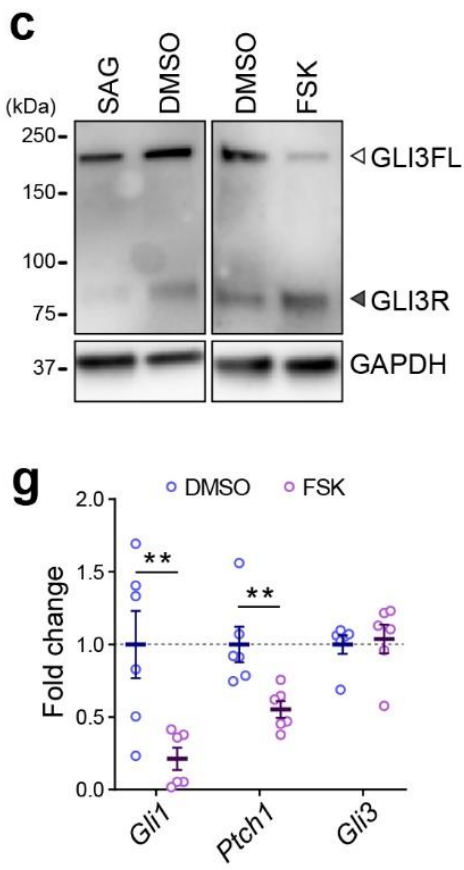

\section{Supplementary Figure 2. GLI3 processing and subcellular localization relies on the primary cilium and regulates Hedgehog signaling target genes.}

a) Representative immunofluorescence pictures showing GLI3 (red) localization in primary cilia stained with both acaTUB (purple) and ARL13B (green) upon SAG (Smoothened agonist) or FSK (forskolin) treatment. DMSO (vehicle) is used as a control. Scale bars, $5 \mu \mathrm{m}$. b) Proportion of DMSO, SAG or FSK treatedmyoblasts that exhibit GLI3 staining in the primary cilium $(n=3)$. c) Immunoblot analysis of GLI3 full-length (GLI3FL) and repressor (GLI3R) in DMSO, SAG or FSK treated-myoblasts. GAPDH is used as a loading control. d) Densitometric analysis of the level of GLI3FL (light gray) and GLI3R (dark gray) relative to GAPDH signals of 5 biological replicates. e) Ratio of GLI3FL/GLI3R relative to GAPDH $(n=5)$. 
f) Expression levels of Gli1 and Ptch1, two Hh target genes, normalized to Ppia and Gapdh, upon SAG treatment, serving as a metric of canonical Hh pathway activation $(n=6)$. g) Expression levels of Gli1 and Ptch1, two Hh target genes, normalized to Ppia and Gapdh, upon FSK treatment, showing the inhibition of canonical Hh signaling $(n=6)$. h) Immunblot analysis of GLI3 and IFT88 confirming the efficiency of Gli3 and Ift88 knockdown in primary myoblasts. i) Densitometric analysis of the level of GLI3FL (light gray) and GLI3R (dark gray) relative to GAPDH signals of 4 biological replicates. j) Ratio of GLI3FL/GLI3R relative to GAPDH $(n=4)$. k) Expression of Gli1, Ptch1, Gli3 and Ift88 normalized to Ppia and Gapdh in primary myoblasts, 48h after treatment with siRNA for Gli3 (siGli3) or with a non-target control (siCtr) $(\mathrm{n}=5)$. I) Expression of Gli1, Ptch1, Gli3 and Ift88 normalized to Ppia and Gapdh in primary myoblasts, 48h after treatment with siRNA for Ift88 (silft88) or with a non-target control (siCtr) $(\mathrm{n}=5)$.

Scale bars, $10 \mu \mathrm{m}$; Error bars, SEM; ${ }^{*} p<0.5,{ }^{* *} p<0.01,{ }^{* * *} p<0.001$. 
a

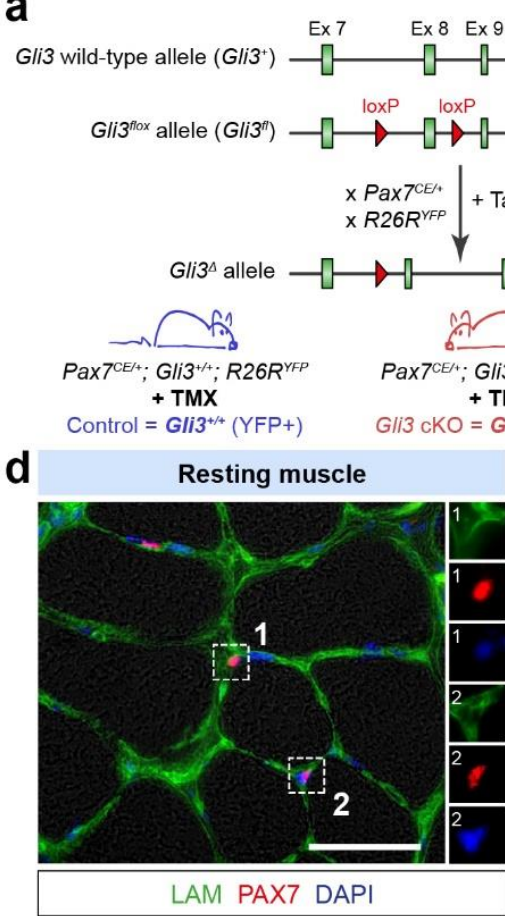

b

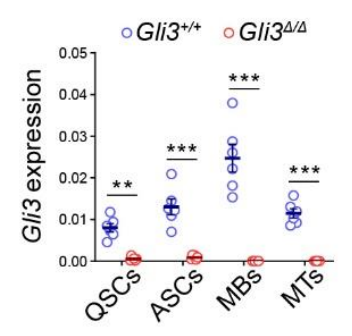

e f

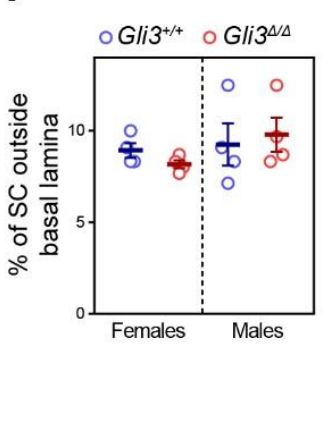

C
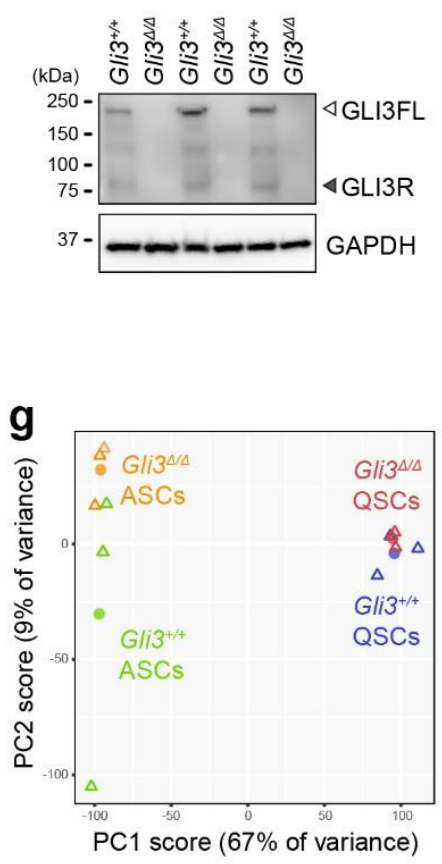

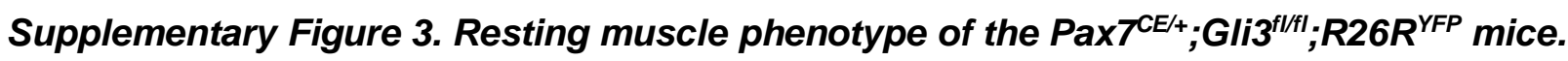

a) The exon 8 of the Gli3 floxed allele (Gli3t) is flanked by two loxP sites, allowing for its tamoxifen (TMX)inducible recombination by the CreER recombinase. The CreER inserted downstream the Pax 7 stop codon $\left(P a x 7^{C E /+}\right.$ ) allows endogenous PAX7 expression while permitting specific ablation of Gli3 in satellite cells upon TMX treatment. In addition, the $R 26 R^{Y F P}$ allele was added allowing for tracing the satellite cell (SC)

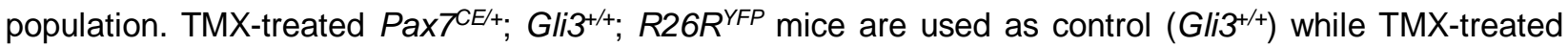
Pax $7^{C E /+}$; Gli3 ${ }^{f / f f ;}$; $26 R^{Y F P}$ mice where SCs will be conditionally knocked-out for Gli3 are referred as Gli3 ${ }^{N / \Delta}$. b) Expression analysis by RT-qPCR of Gli3 normalized to Ppia and Rps18 in quiescent (QSCs) and activated (ASCs) satellite cells, primary myoblasts (MBs), and 3 days differentiated myotubes $(M T s)(n=6)$. c) Western blotting for GLI3 confirming the knockout efficiency in primary myoblasts. GAPDH is used as a loading control ( $\mathrm{n}=3$ biological samples). d) Immunofluorescence for PAX7 (red) and LAMININ (LAM, green) showing 1) a satellite cell within its niche and 2) a satellite cell surrounded by the basal lamina outside its niche. e) Proportion of satellite cells found outside the basal lamina in Gli3+/+ and Gli3 ${ }^{\Delta / \Delta}$ resting TA muscles ( $n=6,3$ males and 3 females for each genotype). f) Proportion of SCs found outside the basal

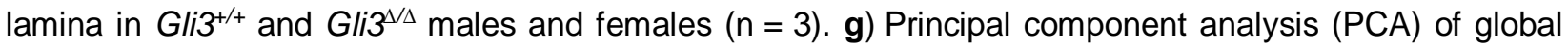
transcriptomes of $\mathrm{Gli3}^{+/+}$and Gli3 ${ }^{\mathrm{N} \Delta}$ quiescent satellite cells (QSCs) and activated satellite cells (ASCs). Each triangle represents a biological replicate. Each dot represents the mean of 3 biological samples $(n=3$ males for each condition and genotype). 


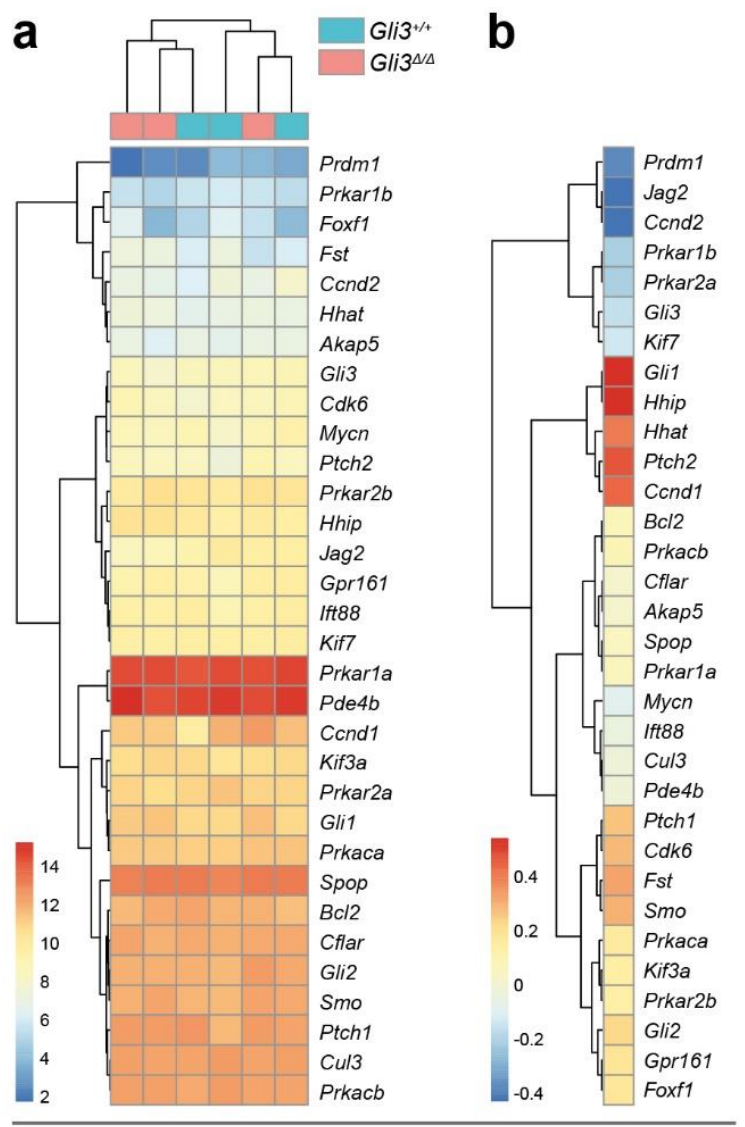

Quiescent satellite cells

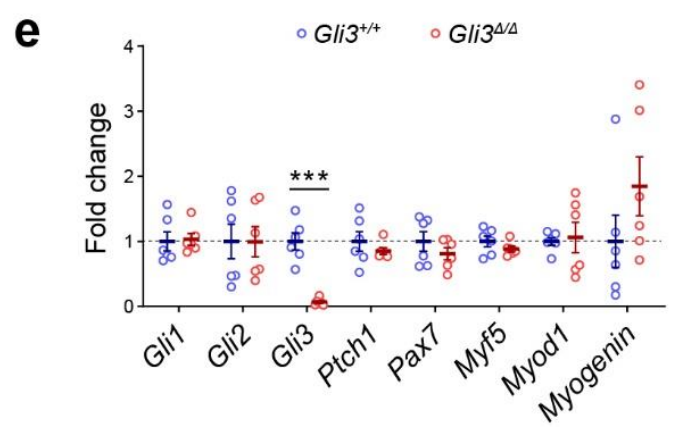

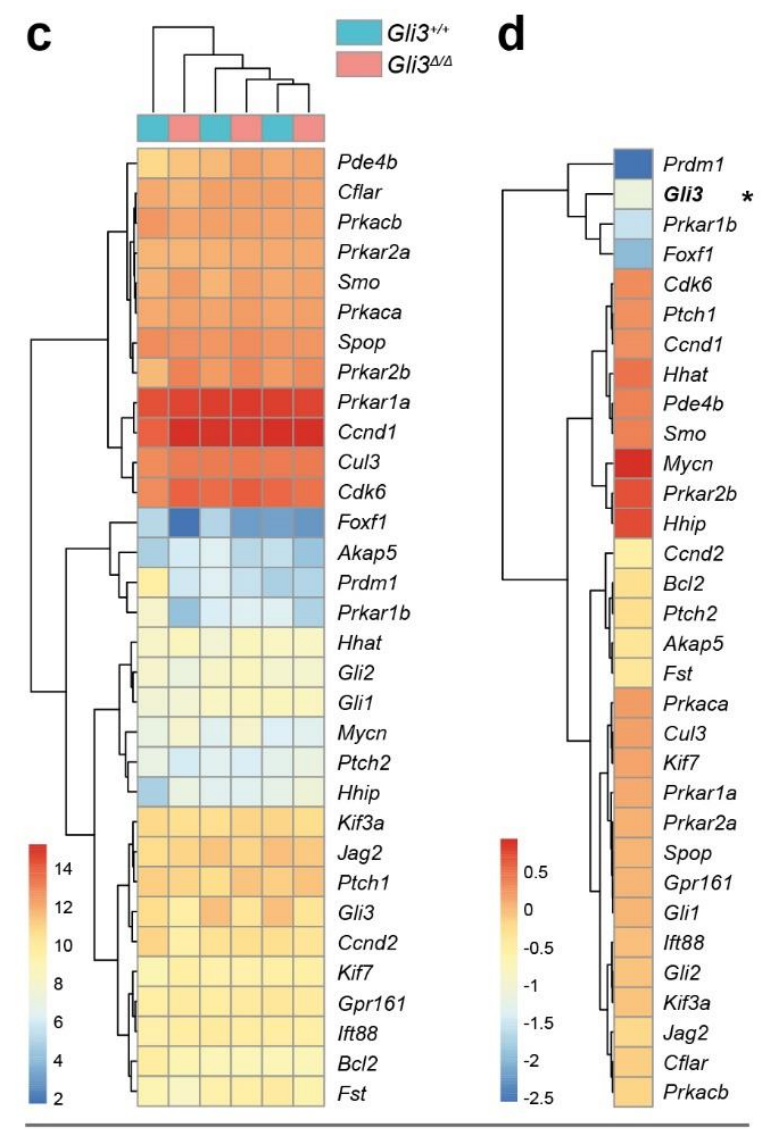

Activated satellite cells
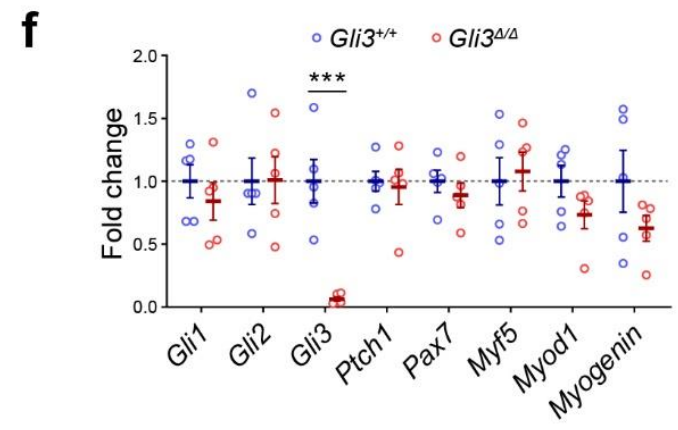

\section{Supplementary Figure 4. Genes of canonical Hedgehog signaling does not vary upon Gli3 deletion in QSCs and ASCs.}

a) Heatmap from normalized and log2 transformed expression matrix of components and target genes of canonical Hedgehog signaling in Gli3 ${ }^{\Delta / \Delta}$ and $\mathrm{Gli}^{+/+}$QSCs ( $n=3$ males). b) Heatmap showing fold change values of canonical Hedgehog signaling genes in Gli3 ${ }^{\Delta / \Delta}$ compared to Gli3 ${ }^{+/+}$QSCs. c) Heatmap from normalized and log2 transformed expression matrix of components and target genes of canonical Hedgehog signaling in Gli3 ${ }^{\Delta / \Delta}$ and $\mathrm{Gli}^{+/+}$ASCs ( $\mathrm{n}=3$ males). d) Heatmap showing fold change values of canonical Hedgehog signaling genes in Gli3 ${ }^{\mathrm{N} \Delta}$ compared to Gli3 ${ }^{+/+}$ASCs. Only Gli3 (in bold, with a star) is significantly down-regulated with a padj <0.05. e) RT-qPCR analysis of Gli1-3, Ptch1, Pax7, Myf5, Myod1 and myogenin normalized to Ppia and Gapdh in Gli3 ${ }^{\Delta / \Delta}$ and Gli3+/+ QSCs $(\mathrm{n}=5)$. f) RT-qPCR analysis of Gli1-3, Ptch1, Pax7, Myf5, Myod1 and myogenin normalized to Ppia and Gapdh in Gli3 ${ }^{\Delta / \Delta}$ and Gli3 ${ }^{+/+}$ASCs $(n=5)$. 


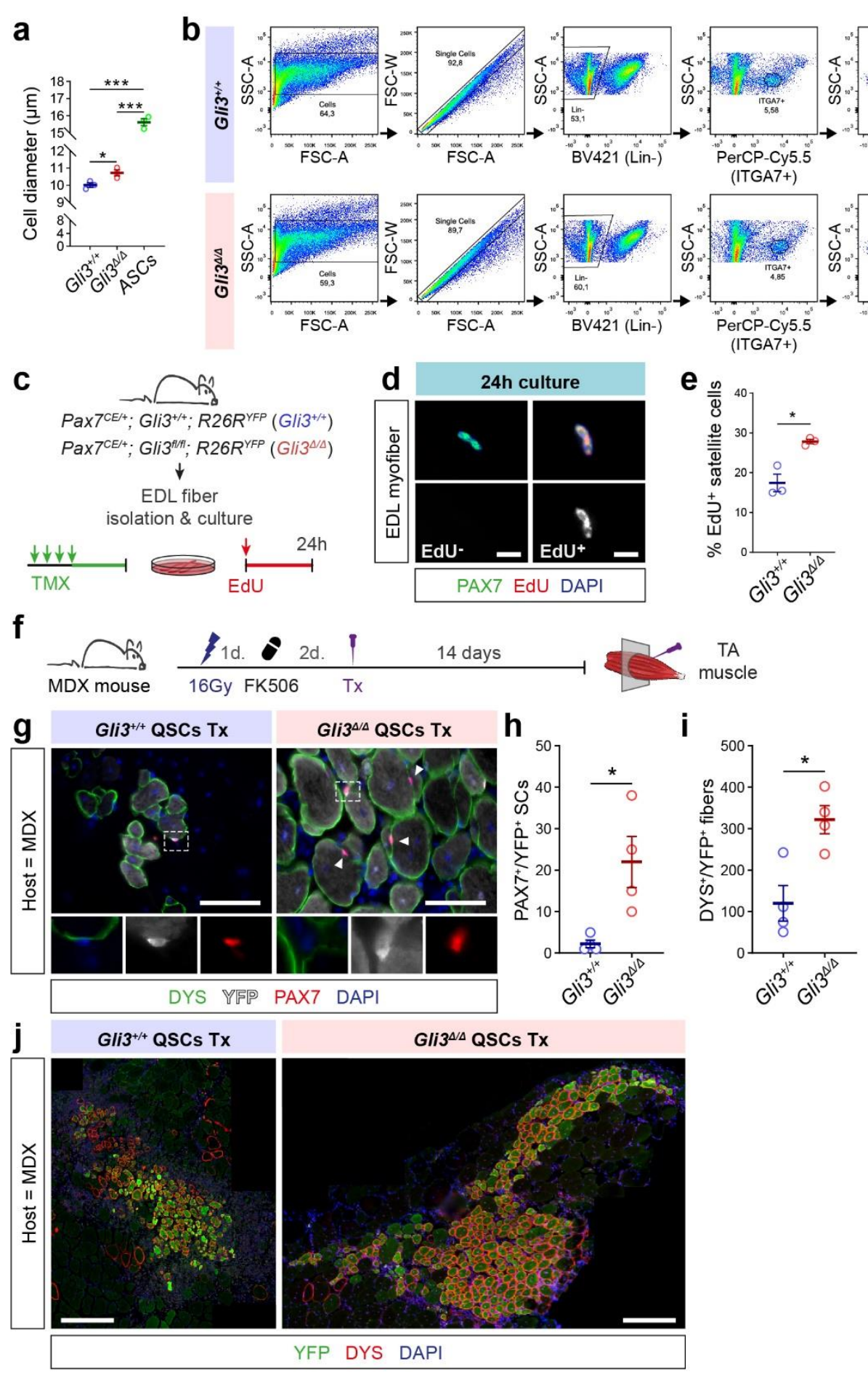

\section{Supplementary Figure 5. Gli3 ${ }^{\Delta \Delta}$ satellite cells exhibit $G_{A l e r t}$ features, enter the cell cycle faster than $\mathrm{Gli3}^{+/+}$satellite cells and engraft better.}

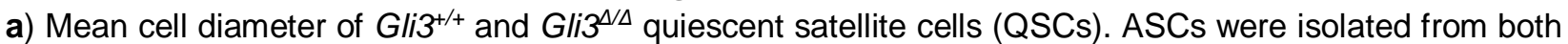
Gli3 $3^{++}$and Gli $3^{\Delta / \Delta}$ mice as they do not exhibit differences ( $n=3$ males). b) Flow cytometry gating strategy

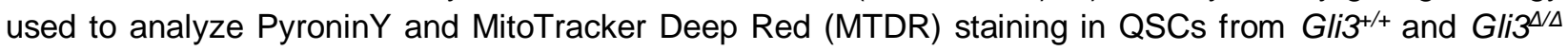
mice. c) Experimental procedure followed to analyze the cell cycle entry of satellite cells from freshly isolated 
myofibers from Gli3 ${ }^{+/+}$and Gli3 ${ }^{\Delta / \Delta}$ mice. d) Representative immunofluorescence of satellite cells (PAX7, green) that have incorporated $\mathrm{EdU}\left(\mathrm{EdU}^{+}\right.$, red) or not (EdU') on 24h-cultured myofibers. Nuclei are stained with DAPI (blue). e) Proportions of EdU ${ }^{+}$satellite cells $40 \mathrm{~h}$ after isolation from $\mathrm{Gli}^{+/+}$and $\mathrm{Gli}^{\mathrm{N} \Delta}$ mice $(\mathrm{n}=3$ males). Scale bars, $10 \mu \mathrm{m}$. f) 10,000 freshly isolated quiescent satellite cells (QSCs) from $\mathrm{Gli}^{+/+}$and Gli3 $3^{\mathrm{V} \Delta}$ mice were transplanted into TA muscles of 16Gy-irradiated and FK506-immunocompromised MDX mice. g) Immunofluorescence picture of transverse sections of MDX host TA muscle 14 days post-engraftment. Both satellite cells (PAX7, red) and myofibers (DYS, green) from the donors express YFP (grey). DAPI stains the nuclei. Scale bars, $50 \mu \mathrm{m}$. h) Number of $\mathrm{PAX7} 7^{+} / \mathrm{YFP}^{+}$satellite cells per transverse sections of MDX transplanted muscles. i) Number of $\mathrm{DYS}^{+} / \mathrm{YFP}^{+}$myofibers per transverse sections of MDX transplanted muscles. j) Representative immunofluorescence picture showing the size of the engrafted area of MDX muscle 14 days following the transplantation of $10^{4} \mathrm{Gli}^{+/+}$or Gli3 ${ }^{\mathrm{N} / \Delta}$ quiescent satellite cells (QSCs). Donor myofibers express both DYS (red) and YFP (green). DAPI stains the nuclei. Scale bars, $500 \mu \mathrm{m}$. Unless indicated otherwise, $\mathrm{n}=3 \mathrm{Gli}^{+/+}$males and $\geq 3$ Gli3 ${ }^{\Delta / \Delta}$ males; Error bars, SEM; ${ }^{*} p<0.05$; ${ }^{\star \star \star *} p<0.001$. 


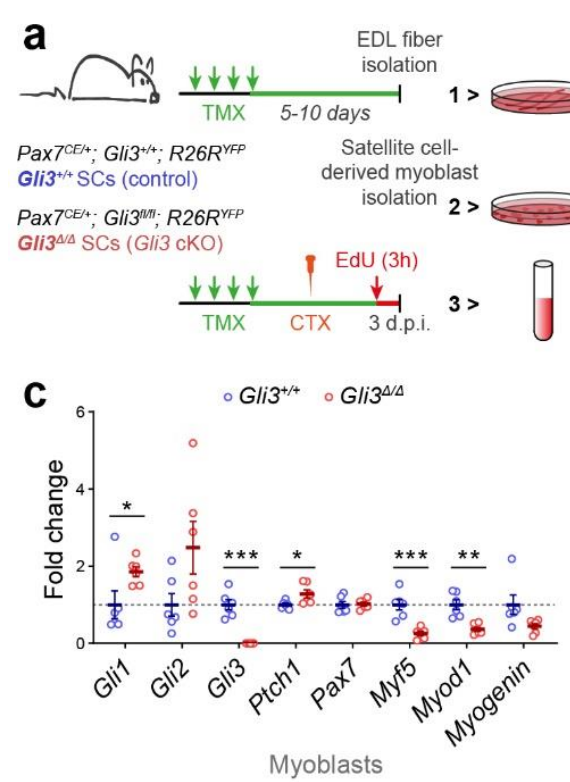
culture

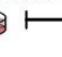

Myoblast culture

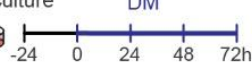

Flow cytometry

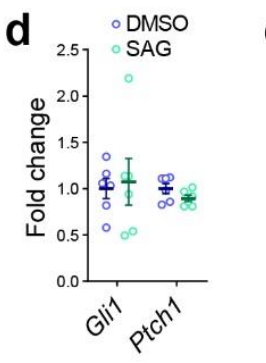

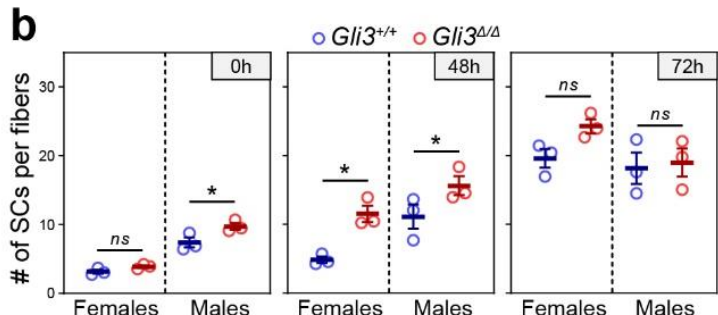
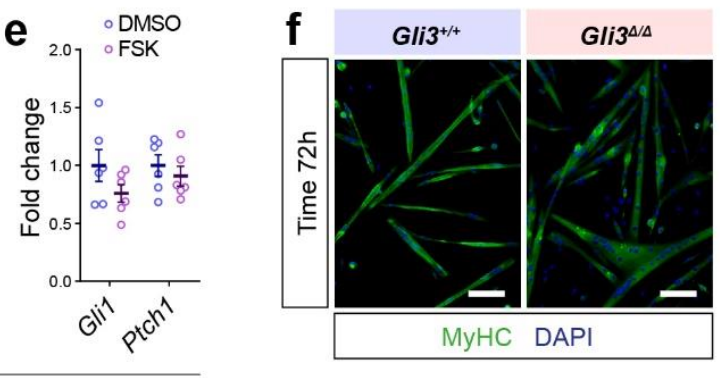

Gli3 $3^{\Delta / \Delta}$ myoblasts

\section{Supplementary Figure 6. Phenotypic characterization of $\mathrm{Gli3}^{+/+}$and $\mathrm{Gli3}^{\mathrm{V} \Delta}$ activated satellite cells and primary myoblasts.}

a) Experimental design. 1> Single EDL myofibers are isolated from tamoxifen-treated

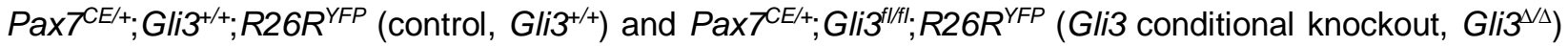
mice and cultured for $48 \mathrm{~h}$ and $72 \mathrm{~h}$ to follow satellite cell proliferation and differentiation. $2>$ Satellite cellderived myoblasts were cultured in proliferating conditions and differentiated for $72 \mathrm{~h}$ in differentiation medium (DM). 3> TA and gastrocnemius (GA) muscle injury was induced by cardiotoxin (CTX) injection. 3 days post-injury ( 3 d.p.i.), activated satellite cells (ASCs) were FACS-isolated and processed for RNA extraction (RNA-sequencing and qPCR). b) Quantification of the number of satellite cells per myofiber immediately after isolation (0h), or after $48 \mathrm{~h}$ and $72 \mathrm{~h}$ of culture $(\mathrm{n}=3$ males and 3 females for each genotype). c) RT-qPCR analysis of Gli1-3, Ptch1, Pax7, Myf5, Myod1 and myogenin normalized to Ppia and Gapdh in Gli3 ${ }^{\mathrm{V} \Delta}$ and Gli3 ${ }^{+/+}$proliferating primary myoblasts $(\mathrm{n}=6)$. d) Expression of Gli1 and Ptch1 normalized to Ppia and Gapdh, in Gli3 ${ }^{\Delta / \Delta}$ myoblasts upon SAG treatment $(\mathrm{n}=6)$. e) Expression of Gli1 and Ptch1 normalized to Ppia and Gapdh, in Gli3 ${ }^{\Delta / \Delta}$ myoblasts upon FSK treatment $(n=6)$. f) Immunostaining of myosin heavy chains (MyHC, green) of $72 \mathrm{~h}$-differentiated $\mathrm{Gli} 3^{\Delta / \Delta}$ and $\mathrm{Gli}^{+/+}$myoblasts. Nuclei are counterstained with DAPI (blue). Scale bar, 100 $\mu$ m.

Error bars, SEM; ${ }^{*} p<0.05 ;{ }^{* *} p<0.01 ;{ }^{* * *} p<0.001$. 

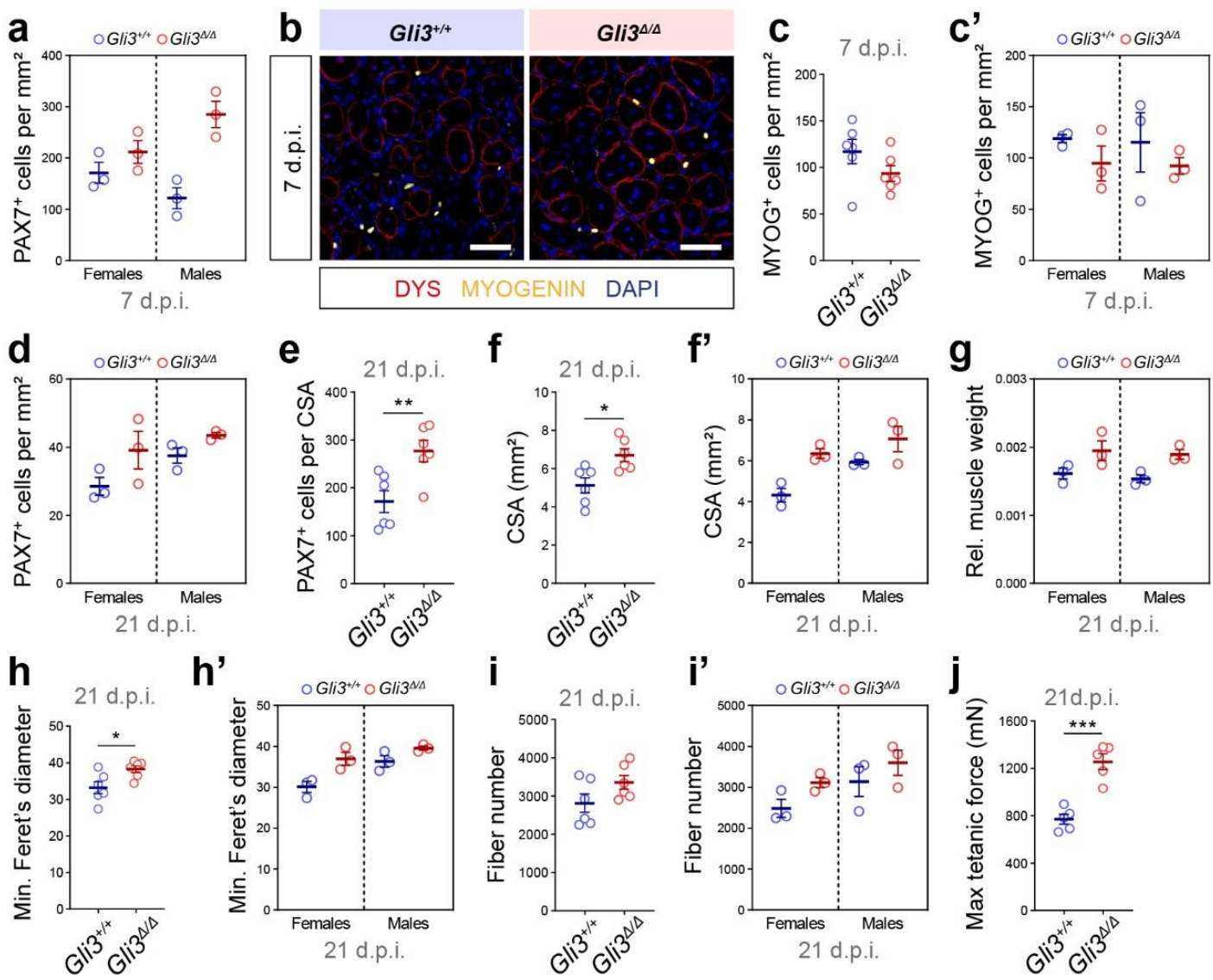

h'
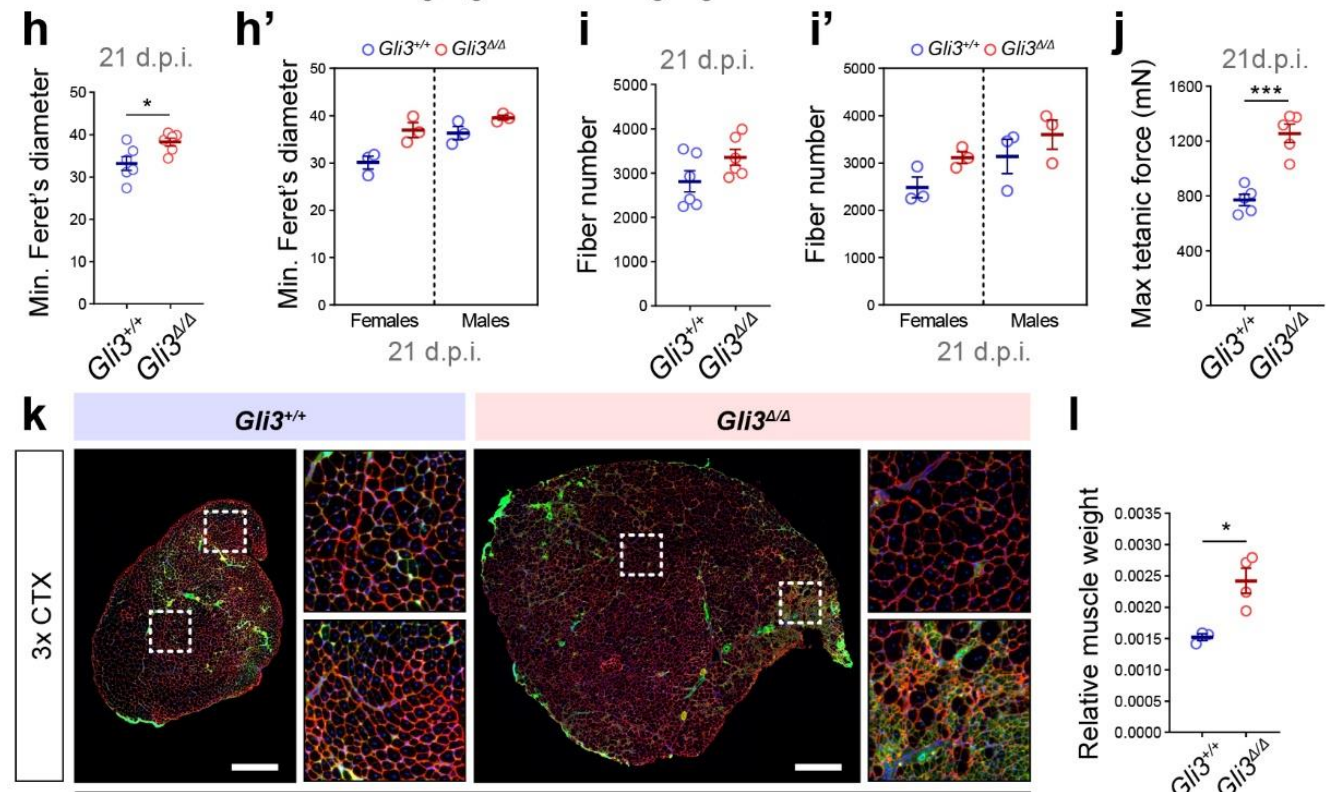

$\mathrm{Gli3}^{\Delta / \Delta}$
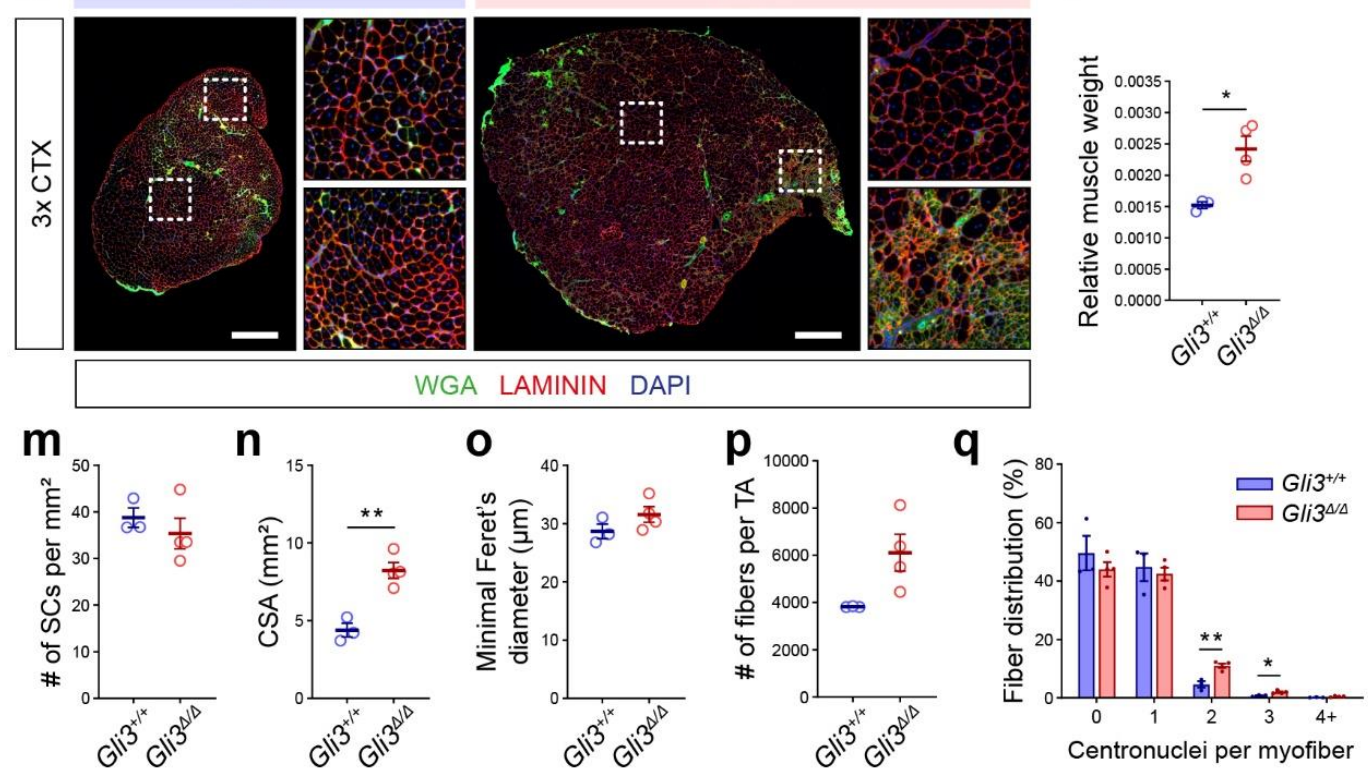

\section{Supplementary Figure 7. Phenotype of $\mathrm{Gli3}^{+/+}$and $\mathrm{Gli3}^{\mathrm{N} \Delta}$ muscle following a single and} triple cardiotoxin-injuries.

a) Quantification of PAX7 $7^{+}$cells per $\mathrm{mm}^{2} \mathrm{TA}$ section of $\mathrm{Gli3^{+/+ }}$ and $\mathrm{Gli} 3^{\mathrm{N} \Delta}$ female and male mice at 7 d.p.i.

b) Immunostaining of MYOGENIN (yellow) at 7 d.p.i. showing the differentiated muscle cells and DYSTROPHIN (DYS, red) delineating the regenerating myofibers. DAPI stains the nuclei (blue). 
c) Quantification of $\mathrm{MYOG}^{+}$cells per $\mathrm{mm}^{2} \mathrm{TA}$ section of all $\mathrm{Gli} 3^{+/+}$and Gli3 ${ }^{\mathrm{V} \Delta}$ mice at 7 d.p.i. and $\mathbf{c}^{\prime}$ ) in females and males. d) Quantification of PAX7 $7^{+}$cells per $\mathrm{mm}^{2} \mathrm{TA}$ section $\mathrm{Gli3^{+/+ }}$ and $\mathrm{Gli} 3^{\mathrm{\Delta} \Delta}$ female and male mice at 21 d.p.i. e) Quantification of PAX7 $7^{+}$cells per TA cross-sectional area (CSA). f) CSA of TA section of all $G / 3^{+/+}$and $G$ li3 $3^{\Delta / \Delta}$ mice at 21 d.p.i. and f') in females and males. g) Relative muscle weight of $\mathrm{Gli3}^{+/+}$ and $G / i 3^{\Delta / \Delta}$ female and male mice at 21 d.p.i. h) Minimal Feret's diameter of all $\mathrm{Gli3}^{+++}$and $\mathrm{Gli} 3^{\Delta / \Delta}$ mice at

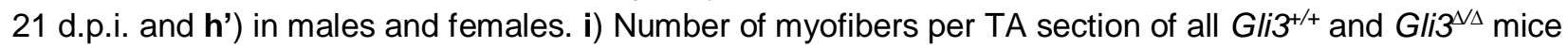
at 21 d.p.i. and i') in males and females. j) Maximum tetanic force of TA muscles of $\mathrm{Gli}^{+/+}$and $\mathrm{Gli} 3^{\mathrm{\Delta} \Delta}$ mice at 21 d.p.i. ( $n=5$ males). Unless indicated otherwise, $n=6$ ( 3 males and 3 females for each genotype); Scale bars, $50 \mu \mathrm{m}$; Error bars, SEM; ${ }^{*} p<0.05 ;{ }^{* *} p<0.01 ;{ }^{* \star *} p<0.001$.

k) Representative immunofluorescence picture of $\mathrm{Gli}^{+/+}$or $\mathrm{Gli} 3^{\mathrm{N} \Delta}$ regenerated muscle following a triple injury (3x CTX). LAMININ (red) and WGA (green) delineate the myofibers. DAPI stains the nuclei. I) TA muscle weight normalized to total body weight of $\mathrm{Gli}^{+/+}$and Gli3 ${ }^{\Delta / \Delta}$ mice following a triple injury. m) Quantification of satellite cells per mm $\mathrm{mm}^{2} 3$-injured TA muscle section of $\mathrm{Gli}^{+/+}$and Gli3 ${ }^{\Delta / \Delta}$ mice. $\mathbf{n}$ ) Crosssectional area (CSA) of 3x-injured TA muscle section of $\mathrm{Gli}^{+/+}$and $\mathrm{Gli} 3^{\mathrm{N} /}$ mice. o) Minimal Feret's diameter of regenerated myofibers of $3 x$-injured $G / 3^{+/+}$and Gli3 ${ }^{\Delta / \Delta}$ mice. p) Number of myofibers per TA section of $\mathrm{Gli}^{+++}$and Gli3 ${ }^{\Delta \Delta}$ mice after a triple injury. q) Distribution of regenerated myofibers according to their number of centrally located nuclei (centronuclei) of $3 x$-injured Gli3 ${ }^{+/+}$and Gli3 $3^{\mathrm{V} \Delta}$ mice.

For the triple injuries ( $3 x \mathrm{CTX}), \mathrm{n}=3 \mathrm{Gli3^{+/+ }}$ males and 4 Gli3 ${ }^{\Delta / \Delta}$ males; Scale bars, $500 \mu \mathrm{m}$; Error bars, SEM; ${ }^{*} p<0.05 ;{ }^{* *} p<0.01$. 